DOI 10.4171/JEMS/529

Hiroshi Matano $\cdot$ Fabio Punzo · Alberto Tesei

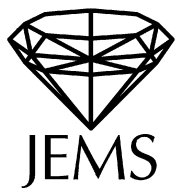

\title{
Front propagation for nonlinear diffusion equations on the hyperbolic space
}

Received August 28, 2012

\begin{abstract}
We study the Cauchy problem in the hyperbolic space $\mathbb{H}^{n}(n \geq 2)$ for the semilinear heat equation with forcing term, which is either of KPP type or of Allen-Cahn type. Propagation and extinction of solutions, asymptotical speed of propagation and asymptotical symmetry of solutions are addressed. With respect to the corresponding problem in the Euclidean space $\mathbb{R}^{n}$ new phenomena arise, which depend on the properties of the diffusion process in $\mathbb{H}^{n}$. We also investigate a family of travelling wave solutions, named horospheric waves, which have properties similar to those of plane waves in $\mathbb{R}^{n}$.
\end{abstract}

Keywords. Semilinear parabolic equations, hyperbolic space, extinction and propagation, asymptotical symmetry of solutions, horospheric waves

\section{Introduction}

In this paper we study the Cauchy problem for the semilinear parabolic equation

$$
\begin{cases}\frac{\partial u}{\partial t}=\Delta_{H} u+f(u) & \text { in } \mathbb{H}^{n} \times \mathbb{R}_{+}, \\ u=u_{0} & \text { in } \mathbb{H}^{n} \times\{0\},\end{cases}
$$

in the hyperbolic space $\mathbb{H}^{n}(n \geq 2)$. Here $\Delta_{H}$ denotes the Laplace-Beltrami operator in $\mathbb{H}^{n}$ and $\mathbb{R}_{+} \equiv(0, \infty)$.

Concerning the Cauchy data function $u_{0}$, the following assumption is always made:

$$
u_{0} \text { continuous in } \mathbb{H}^{n}, \quad 0 \leq u_{0}(x) \leq 1 \quad \text { for any } x \in \mathbb{H}^{n} .
$$

As for the function $f$, we always assume

$$
f \in C^{1}([0,1]), \quad f(0)=f(1)=0 .
$$

H. Matano: Department of Mathematics, Graduate School of Mathematical Sciences, University of Tokyo, 3-8-1 Komaba, Meguro-ku, Tokyo 153-8902, Japan; e-mail: matano@ms.u-tokyo.ac.jp F. Punzo: Dipartimento di Matematica "F. Enriques", Università degli Studi di Milano, via C. Saldini 50, I-20133 Milano, Italy; e-mail: fabio.punzo@unimi.it

A. Tesei: Dipartimento di Matematica "G. Castelnuovo", Università di Roma "La Sapienza", P.le A. Moro 5, I-00185 Roma, Italy; e-mail: tesei@mat.uniroma1.it

Mathematics Subject Classification (2010): 35K57, 35B40 
Moreover, either

$$
f^{\prime}(0)>0, \quad f(u)>0 \quad \text { for any } u \in(0,1)
$$

or

$$
\left\{\begin{array}{l}
\text { (i) there exist } a \in(0,1) \text { such that } \\
f(u)<0 \text { for any } u \in(0, a), f(u)>0 \text { for any } u \in(a, 1) \text {; } \\
\text { (ii) } f^{\prime}(0)<0, \int_{0}^{1} f(u) d u>0 .
\end{array}\right.
$$

Following a common terminology, the function $f$ is said to be of KPP type if it satisfies assumption $\left(H_{1}\right)$, and of Allen-Cahn type if $\left(H_{2}\right)$ holds.

Solutions of problem (1.1) are always meant in the classical sense. By assumptions (A), $\left(H_{0}\right)$ and comparison results [17], every solution $u$ of problem (1.1) satisfies the inequality

$$
0 \leq u(x, t) \leq 1 \quad \text { for any }(x, t) \in \mathbb{H}^{n} \times \mathbb{R}_{+} .
$$

Under assumptions $(A)$ and $\left(H_{0}\right)$ a unique solution of problem (1.1) is easily seen to exist. In fact, existence follows by the a priori estimate (1.2) and standard compactness arguments, and uniqueness by [17, Theorem 3.1].

The counterpart of problem (1.1) in $\mathbb{R}^{n}$, namely

$$
\begin{cases}\frac{\partial u}{\partial t}=\Delta u+f(u) & \text { in } \mathbb{R}^{n} \times \mathbb{R}_{+}, \\ u=u_{0} & \text { in } \mathbb{R}^{n} \times\{0\},\end{cases}
$$

has been widely investigated (in particular, see [2, 3, 6, 9, 13]). Let us recall some wellknown results (see [3] for details).

(a) If the forcing term $f$ is of KPP type, then propagation always occurs, that is,

$$
\lim _{t \rightarrow \infty} u(x, t)=1 \quad \text { uniformly on compact subsets of } \mathbb{R}^{n}
$$

for every solution $u \not \equiv 0$ of problem (1.3). This follows from the so-called "hair-trigger effect": if $f$ satisfies $\left(H_{0}\right)$ and

(i) there exists $a \in(0,1]$ such that $f(u)>0$ for any $u \in(0, a)$;

(ii) $\liminf _{u \rightarrow 0^{+}} u^{-(1+2 / n)} f(u)>0$,

then for every solution $u \not \equiv 0$ of problem (1.3),

$$
\liminf _{t \rightarrow \infty} u(x, t) \geq a \quad \text { uniformly on compact subsets of } \mathbb{R}^{n} .
$$

Clearly, assumption $\left(H_{1}\right)$ implies $(H T)$ with $a=1$, thus (1.4) follows. Observe that the exponent $p=1+2 / n$ in $(H T)$ (ii) is the Fujita exponent of problem (1.3) with $f(u)=u^{p}($ see $[10])$.

More generally, assumption $(H T)$ is satisfied if $(H T)(\mathrm{i})$ holds and

$$
\liminf _{u \rightarrow 0^{+}} u^{-p} f(u)>0 \quad \text { for some } p \in(1,1+2 / n),
$$


thus (1.5) follows also in this case. On the other hand, if

$$
\limsup _{u \rightarrow 0^{+}} u^{-p} f(u)<\infty \quad \text { for some } p>1+2 / n
$$

and $u_{0}$ is small enough, then extinction occurs, that is,

$$
\lim _{t \rightarrow \infty} u(x, t)=0 \quad \text { uniformly in } \mathbb{R}^{n} .
$$

(b) If $f$ is of Allen-Cahn type, there is a "threshold effect". In fact, there is extinction (that is, equality (1.7) holds) if the initial data function $u_{0}$ is suitably small; instead, there is propagation (that is, equality (1.4) holds true) if $u_{0}$ is sufficiently large.

(c) Both for KPP and for Allen-Cahn there exists an asymptotic speed of propagation $c_{0}>0$, which is uniquely determined by the following properties:

(i) No solution of problem (1.3) with $u_{0}$ having compact support can propagate with speed greater than $c_{0}$. In fact, for any $c>c_{0}$ and $y \in \mathbb{R}^{n}$,

$$
\lim _{t \rightarrow \infty} \sup _{|x-y|>c t} u(x, t)=0 .
$$

(ii) If a solution of problem (1.3) propagates, then its speed is no smaller than $c_{0}$. In fact, if

$$
\liminf _{t \rightarrow \infty} u(x, t) \geq a \quad \text { uniformly on compact subsets of } \mathbb{R}^{n}
$$

for some $a \in(0,1]$, then for any $c<c_{0}$ and $y \in \mathbb{R}^{n}$,

$$
\liminf _{t \rightarrow \infty} \inf _{|x-y|<c t} u(x, t) \geq a \text {. }
$$

Observe that the asymptotic speed of propagation $c_{0}$ only depends on the forcing term $f$. In fact, its definition relies on a detailed investigation of the ordinary differential equation

$$
q^{\prime \prime}+\kappa q^{\prime}+f(q)=0 \quad \text { in } \mathbb{R} \quad(\kappa \in \mathbb{R})
$$

(in this connection, see Proposition 3.1 below). Equation (1.8) arises when seeking plane wave solutions of the equation

$$
\frac{\partial u}{\partial t}=\Delta u+f(u) \quad \text { in } \mathbb{R}^{n} \times \mathbb{R}_{+},
$$

that is, solutions of equation (1.9) of the form

$$
u(x, t)=q(\langle x, v\rangle-\kappa t) \quad\left(x \in \mathbb{R}^{n}, t>0\right),
$$

where $q$ is a real function, $v \in \mathbb{R}^{n}$ is a fixed unit direction, $\kappa \in \mathbb{R}$ and $\langle x, v\rangle:=\sum_{i=1}^{n} x_{i} v_{i}$.

Under the additional assumption

$$
\sup _{u \in(0,1]} \frac{f(u)}{u}=f^{\prime}(0)
$$

we have $c_{0}=2 \sqrt{f^{\prime}(0)}$ (see [3, Proposition 4.2 and following remarks]). 
In this paper we prove results for problem (1.1) which are analogous to those above for problem (1.3), yet exhibit remarkably novel features compared to the Euclidean case. In fact, the following holds.

$\left(\mathrm{a}^{\prime}\right)$ If the function $f$ is of KPP type, under the additional assumption $\left(H_{1}^{\prime}\right)$ we prove the existence of a new threshold effect. In fact, extinction prevails if

$$
c_{0}=2 \sqrt{f^{\prime}(0)}<n-1
$$

and $u_{0}$ has compact support, whereas there is propagation if $c_{0}>n-1$ (see Theorem 3.2). Therefore, in contrast to the Euclidean case, in the hyperbolic space we can have extinction even in the KPP case, depending on the sign of the difference $c_{0}-(n-1)$.

This implies that no hair-trigger effect holds in $\mathbb{H}^{n}$, for otherwise no extinction could arise for the KPP case, which is a contradiction when $c_{0}<n-1$. In this connection, observe that the proof of the hair-trigger effect in $\mathbb{R}^{n}$ given in [3] relies on the Fujita phenomenon, whereas it is known that the Fujita exponent of problem (1.1) is $p=1$ (see [4]) -namely, global solutions of problem (1.1) with $f(u)=u^{p}$ exist for any $p>1$ if $u_{0}$ is sufficiently small. This remark also explains Theorem 3.3, which shows that every solution of problem (1.1) is extinct if

$$
\limsup _{u \rightarrow 0^{+}} u^{-p} f(u)<\infty \quad \text { for some } p>1
$$

and $u_{0}$ is sufficiently small (condition (1.11) should be compared with (1.6) of the Euclidean case).

$\left(\mathrm{b}^{\prime}\right)$ If $f$ is of Allen-Cahn type, we prove extinction if the initial data function $u_{0}$ is sufficiently small, respectively propagation if $u_{0}$ is sufficiently large and $c_{0}>n-1$ (see Theorems 3.4 and 3.5). Hence the sign of the difference $c_{0}-(n-1)$ plays a role also in this case.

$\left(c^{\prime}\right)$ The role of the difference $c_{0}-(n-1)$ becomes clear by addressing the asymptotical speed of propagation of problem (1.1). In fact, in the hyperbolic space this speed (both for the KPP and the Allen-Cahn case) turns out to be $c_{0}-(n-1)$ (see Theorem 3.7).

A first heuristic explanation of the above differences can be given by comparing the expression in polar coordinates $(r, \theta)$ in $\mathbb{R}^{n}$ of equation (1.9) with that in polar geodesic coordinates $(\rho, \theta)$ in $\mathbb{H}^{n}$ of the equation

$$
\frac{\partial u}{\partial t}=\Delta_{H} u+f(u) \quad \text { in } \mathbb{H}^{n} \times \mathbb{R}_{+} .
$$

We have

$$
\frac{\partial u}{\partial t}=\frac{\partial^{2} u}{\partial r^{2}}+\frac{n-1}{r} \frac{\partial u}{\partial r}+\frac{1}{r^{2}} \Delta_{\theta} u+f(u),
$$

respectively

$$
\frac{\partial u}{\partial t}=\frac{\partial^{2} u}{\partial \rho^{2}}+(n-1) \operatorname{coth} \rho \frac{\partial u}{\partial \rho}+\frac{1}{(\sinh \rho)^{2}} \Delta_{\theta} u+f(u)
$$


(see Section 2; here $\Delta_{\theta}$ denotes the Laplace-Beltrami operator on the $(n-1)$-dimensional sphere of $\mathbb{R}^{n}$ ). While the coefficient of the first order term on the right-hand side of (1.13) tends to zero as $r \rightarrow \infty$, the corresponding term in (1.14) tends to $n-1$ as $\rho \rightarrow \infty$. Therefore, in $\mathbb{H}^{n}$ there is a "drift from infinity" which must be overcome to have propagation. If propagation prevails, the resulting speed is $c_{0}-(n-1)$, and not $c_{0}$ as in $\mathbb{R}^{n}$ where the "drift from infinity" is absent. This explains why extinction can arise in $\mathbb{H}^{n}$ even in the KPP case, and why the condition $c_{0}>n-1$ is needed to have propagation in the Allen-Cahn case.

A related viewpoint is that the different geometrical properties of $\mathbb{H}^{n}$ affect diffusion through the spectral properties of the Laplace-Beltrami operator and the growth estimates of its heat kernel. In this connection, observe that by $\left(H_{1}^{\prime}\right)$,

$$
c_{0}=2 \sqrt{f^{\prime}(0)}>n-1 \Leftrightarrow f^{\prime}(0)>\frac{(n-1)^{2}}{4}=: \lambda_{1},
$$

where $\lambda_{1}$ denotes the infimum of the $L^{2}$-spectrum of the operator $-\Delta_{H}$ in $\mathbb{H}^{n}$. Therefore, the important role of the difference $c_{0}-(n-1)$ is related to the fact that the spectrum has positive infimum. In general terms, the positivity of $\lambda_{1}$ makes diffusion "stronger in $\mathbb{H}^{n}$ than in $\mathbb{R}^{n}$ ". This explains why the Fujita exponent is $p=1$ instead of $p=1+2 / n$, and why extinction more easily prevails over propagation than in the Euclidean case.

Let us point out some technical facts to illustrate the above remarks:

- the condition $f^{\prime}(0)>\lambda_{1}$ plays a role when constructing a suitable family of subsolutions to problem (1.1), which is needed to prove propagation for the KPP case (see the proof of Theorem 3.2);

- estimates from above of the heat kernel in $\mathbb{H}^{n}$ are used to exhibit a family of supersolutions to problem (1.1), which is used to prove extinction in the Allen-Cahn case (see the proof of Theorem 3.5);

- the existence of a ground state, that is, of a positive solution of the equation

$$
\Delta_{H} \phi+\lambda_{1} \phi=0 \quad \text { in } \mathbb{H}^{n}
$$

which is radial and infinitesimal as $\rho \rightarrow \infty$, plays an important role to prove extinction when (1.11) holds (see the proof of Theorem 3.3).

Besides the results outlined in $\left(\mathrm{a}^{\prime}\right)-\left(\mathrm{c}^{\prime}\right)$ above, we shall address the asymptotical symmetry of solutions to problem (1.1) (see Theorem 3.7), extending to the present situation some results in [13]. In doing so, we make use of the Alexandrov reflection method, already used in the hyperbolic space in $[14,15]$.

Finally, we introduce and investigate a family of travelling wave solutions of equation (1.12), named horospheric waves, which in some respects are analogous to plane waves in $\mathbb{R}^{n}$. In this connection, observe that in the Poincaré half-space model $\mathbb{U}^{n}$ of the hyperbolic space the horospheres take the form $x_{n}=$ const $\left.\left(x_{1}, \ldots, x_{n}\right) \in \mathbb{U}^{n}\right)$.

Horospheric waves are defined as solutions of problem (1.1) whose level set is for any $t \in \mathbb{R}_{+}$a horosphere of the form

$$
\left\{x \in \mathbb{U}^{n} \mid x_{n}=K e^{-\kappa t}\right\} \quad(K>0, \kappa \in \mathbb{R})
$$

(see Definition 3.1). In Theorem 3.9 we prove that: 
(i) a solution of this kind exists (both for KPP and for Allen-Cahn) if $c_{0}>n-1$;

(ii) in $\mathbb{U}^{n}$ it has the following representation, formally analogous to (1.10) with $v \equiv$ $(0, \ldots, 0,1)$ :

$$
u\left(x_{1}, \ldots, x_{n}, t\right)=q^{*}\left(-\log x_{n}-c^{*} t\right),
$$

where $c^{*}:=c_{0}-(n-1)$ and $q^{*}$ is the solution of the equation

$$
q^{\prime \prime}+c_{0} q^{\prime}+f(q)=0
$$

such that

$$
\lim _{\xi \rightarrow-\infty} q^{*}(\xi)=1, \quad \lim _{\xi \rightarrow \infty} q^{*}(\xi)=0
$$

Interestingly, it can be proven that horospheric waves give an estimate of any solution to problem (1.1), as plane waves do in the Euclidean case (see Theorem 3.10 and subsequent remarks).

\section{Geometrical background}

By definition, the hyperbolic space $\mathbb{H}^{n}$ is the unique simply connected, complete $n$ dimensional Riemannian manifold with sectional curvature equal to -1 . The hyperbolic distance between any two points $x, y \in \mathbb{H}^{n}$ will be denoted by $d(x, y)$. At any fixed point $x_{0} \in \mathbb{H}^{n}$, the inner Riemannian product $\langle\cdot, \cdot\rangle_{H}$ is given by

$$
\langle\xi, \eta\rangle_{H}:=\sum_{i, j=1}^{n} g_{i j} \xi_{i} \eta_{j},
$$

where $\left(g_{i j}\right)$ denotes the Riemannian metric of $\mathbb{H}^{n}$ and the vectors $\xi \equiv\left(\xi_{1}, \ldots, \xi_{n}\right)$, $\eta \equiv\left(\eta_{1}, \ldots, \eta_{n}\right)$ belong to the tangent space $T_{x_{0}}$.

As is well known, in $\mathbb{H}^{n}$ every geodesic is defined on the whole real line; moreover, there exists exactly one geodesic passing through any two different points of $\mathbb{H}^{n}$. A subset $A \subseteq \mathbb{H}^{n}$ is a hyperbolic subspace if it contains the entire geodesic passing through any two of its points (a hyperbolic subspace of codimension 1 is called a hyperbolic hyperplane). Moreover, a subset $K \subseteq \mathbb{H}^{n}$ is convex if for any $x, y \in K$ the geodesic arc joining $x$ to $y$ lies in $K$. By definition, the convex hull of $A \subseteq \mathbb{H}^{n}$ is the smallest convex set of $\mathbb{H}^{n}$ containing $A$.

Let us recall the definition of reflection in a hyperplane $\pi \subseteq \mathbb{H}^{n}$ (see e.g. [14]). First observe that for any given $x \in \mathbb{H}^{n}$ there exists a unique point $x^{*} \in \pi$ such that $d\left(x, x^{*}\right)=\min _{y \in \pi} d(x, y)$. Let $\gamma: \mathbb{R} \rightarrow \mathbb{H}^{n}$ be the unique entire geodesic joining $x$ to $x^{*}$ such that $\gamma(0)=x^{*}, \gamma\left(t_{0}\right)=x$ for some $t_{0}>0$. Then the reflection $R_{\pi}$ in the hyperplane $\pi$ is defined as follows:

$$
R_{\pi}: \mathbb{H}^{n} \rightarrow \mathbb{H}^{n}, \quad R_{\pi}(x):=\gamma\left(-t_{0}\right) \quad\left(x \in \mathbb{H}^{n}\right) .
$$

Denote by $\partial \mathbb{H}^{n}$ the boundary of the hyperbolic space (see [5]); the points of $\partial \mathbb{H}^{n}$ can be regarded as the points at infinity of $\mathbb{H}^{n}$. By definition, a horosphere centered at 
$x_{0} \in \partial \mathbb{H}^{n}$ is a closed hypersurface of $\mathbb{H}^{n}$ orthogonal to all geodesic lines with endpoint $x_{0}$. Observe that, for any fixed $x_{0} \in \partial \mathbb{H}^{n}, \mathbb{H}^{n}$ is the disjoint union of all horospheres with center $x_{0}$.

Let us recall for the convenience of the reader some properties of different models of $\mathbb{H}^{n}$, which will be used in the following (see e.g. $[5,8,12]$ ).

(a) Set $B_{r}:=\left\{x \in \mathbb{R}^{n}|| x \mid<r\right\}(r>0),|\cdot|$ denoting the Euclidean norm of $\mathbb{R}^{n}$. The Poincaré disk model $\mathbb{D}^{n}$ is the unit ball $B_{1}$ endowed with the Riemannian metric

$$
g_{i j}:=\frac{4}{\left(1-|x|^{2}\right)^{2}} \delta_{i j} \quad\left(x \in \mathbb{D}^{n} ; i, j=1, \ldots, n\right) .
$$

Let us mention for further reference that:

(i) geodesics in $\mathbb{D}^{n}$ are either diameters of $\mathbb{D}^{n}$ or circles orthogonal to $\partial \mathbb{D}^{n}$;

(ii) a subset $\pi \subseteq \mathbb{D}^{n}$ is a hyperbolic hyperplane if and only if it is the intersection of $\mathbb{D}^{n}$ either with a hyperplane of $\mathbb{R}^{n}$ through the origin, or with an $(n-1)$-dimensional sphere orthogonal to $\partial \mathbb{D}^{n}$ (see [5]);

(iii) in $\mathbb{D}^{n}$, a horosphere with center $x_{0} \in \partial \mathbb{H}^{n}$ is an $(n-1)$-dimensional sphere of $\mathbb{R}^{n}$ contained in $\mathbb{D}^{n}$ and tangent at $x_{0}$ to $\partial \mathbb{D}^{n}$.

For any fixed point $O \in \mathbb{H}^{n}$ and $x \in \mathbb{H}^{n}$ we can consider the geodesic coordinates $(\rho, \theta)$ of $x$, namely $\rho \equiv \rho(x):=d(x, O)$. In $\mathbb{D}^{n}$, taking $O$ at the origin we have

$$
\rho(x)=\int_{0}^{|x|} \frac{2}{1-s^{2}} d s=\log \left(\frac{1+|x|}{1-|x|}\right)
$$

thus

$$
|x|=\tanh \frac{\rho(x)}{2}, \quad \frac{2}{1-|x|^{2}}=2\left[\cosh \frac{\rho(x)}{2}\right]^{2} \quad\left(x \in \mathbb{D}^{n}\right) .
$$

Therefore, defining

$$
\mathfrak{B}_{r}:=\left\{x \in \mathbb{H}^{n} \mid \rho(x)<r\right\} \quad(r>0),
$$

in $\mathbb{D}^{n}$ we have, for any $r \in(0,1)$,

$$
B_{r}=\mathfrak{B}_{\log \left(\frac{1+r}{1-r}\right)}
$$

Let us recall that in $\mathbb{D}^{n}$ there is an isometry of the form (see [1])

$$
\tau_{y}: \mathbb{D}^{n} \rightarrow \mathbb{D}^{n}, \quad \tau_{y}(x):=\frac{\left(1-|y|^{2}\right)(x-y)-|x-y|^{2} y}{\left(1+|x|^{2}|y|^{2}-2 \sum_{i=1}^{n} x_{i} y_{i}\right)^{2}} \quad\left(x, y \in \mathbb{D}^{n}\right) .
$$

It is easily seen that $\tau_{-y}=\tau_{y}^{-1}\left(y \in \mathbb{D}^{n}\right)$. Moreover,

$$
\left|\tau_{y}(x)\right|=\frac{|x-y|}{1+|x|^{2}|y|^{2}-2 \sum_{i=1}^{n} x_{i} y_{i}} \quad\left(x, y \in \mathbb{D}^{n}\right) .
$$


For any $x, y \in \mathbb{D}^{n}$ we have

$$
d(x, y)=d\left(\tau_{x}(x), \tau_{x}(y)\right)=d\left(0, \tau_{x}(y)\right)=\log \left(\frac{1+\left|\tau_{x}(y)\right|}{1-\left|\tau_{x}(y)\right|}\right) .
$$

Recall that the group of isometries of $\mathbb{D}^{n}$ coincides with the group of conformal mappings of $\mathbb{D}^{n}$ (see [5]). In particular, to prove Theorem 3.7 we shall use a particular class of isometries, namely the reflections in hyperplanes of $\mathbb{H}^{n}$.

The gradient $\nabla_{H} u \equiv\left(\left(\nabla_{H} u\right)_{1}, \ldots,\left(\nabla_{H} u\right)_{n}\right)$ of a function $u \in C^{1}\left(\mathbb{H}^{n}\right)$ is given by

$$
\left(\nabla_{H} u\right)_{i}:=\sum_{j=1}^{n} g^{i j} \frac{\partial u}{\partial x_{j}} \quad(i=1, \ldots, n)
$$

The Laplace-Beltrami operator in $\mathbb{H}^{n}$ of a function $u \in C^{2}\left(\mathbb{H}^{n}\right)$ is

$$
\Delta_{H} u \equiv \frac{1}{\sqrt{g}} \sum_{i=1}^{n} \frac{\partial}{\partial x_{i}}\left(\sqrt{g} \sum_{j=1}^{n} g^{i j} \frac{\partial u}{\partial x_{j}}\right),
$$

where $g:=\operatorname{det}\left(g_{i j}\right), g^{i j}:=\left(g_{i j}\right)^{-1}$. As already mentioned, the infimum of the $L^{2}$ spectrum of the operator $-\Delta_{H}$ in $\mathbb{H}^{n}$ is

$$
\lambda_{1}:=(n-1)^{2} / 4
$$

Moreover,

$$
\lambda_{1}\left(\mathfrak{B}_{r}\right) \searrow \lambda_{1} \quad \text { as } r \rightarrow \infty
$$

(see [11]), where $\lambda_{1}\left(\mathfrak{B}_{r}\right)$ is the first eigenvalue for $-\Delta_{H}$ in $\mathfrak{B}_{r}$ with Dirichlet zero boundary conditions:

$$
\begin{cases}\Delta_{H} \varphi_{1}+\lambda_{1}\left(\mathfrak{B}_{r}\right) \varphi_{1}=0 & \text { in } \mathfrak{B}_{r} \\ \varphi_{1}=0 & \text { on } \partial \mathfrak{B}_{r}(r>0),\end{cases}
$$

and $\varphi_{1}=\varphi_{1}(\rho)>0$ denotes the corresponding eigenfunction.

Let us recall that $\Delta_{H}$ commutes with isometries $\tau$ of $\mathbb{H}^{n}$ (see e.g. [14]). Hence, if $u$ is a solution of the equation

$$
\frac{\partial u}{\partial t}=\Delta_{H} u+f(u) \quad \text { in } \mathbb{H}^{n}
$$

then $v:=u \circ \tau$ satisfies

$$
\frac{\partial v}{\partial t}=\Delta_{H} v+f(v) \quad \text { in } \mathbb{H}^{n} .
$$

By (2.3) and (2.8), the Laplace-Beltrami operator in $\mathbb{D}^{n}$ has the expression

$$
\Delta_{H} u=\frac{1}{4}\left(1-|x|^{2}\right)^{2} \sum_{i=1}^{n} \frac{\partial^{2} u}{\partial x_{i}^{2}}+\frac{n-2}{2}\left(1-|x|^{2}\right) \sum_{i=1}^{n} x_{i} \frac{\partial u}{\partial x_{i}},
$$


thus it can be regarded as a linear elliptic operator on $B_{1} \subseteq \mathbb{R}^{n}$ with bounded coefficients degenerating at the boundary $\partial B_{1}$. Using polar geodesic coordinates $(\rho, \theta)$ in $\mathbb{D}^{n}$, so that

$$
d s^{2}:=\sum_{i, j=1}^{n} g_{i j} d x_{i} d x_{j}=d \rho^{2}+(\sinh \rho)^{2} d \theta
$$

we obtain

$$
\Delta_{H} u=\frac{\partial^{2} u}{\partial \rho^{2}}+(n-1) \operatorname{coth} \rho \frac{\partial u}{\partial \rho}+\frac{1}{(\sinh \rho)^{2}} \Delta_{\theta} u,
$$

$\Delta_{\theta}$ being the Laplace-Beltrami operator on the $(n-1)$-dimensional sphere of $\mathbb{R}^{n}$.

In $\mathbb{H}^{n}$ the heat kernel $G: \mathbb{H}^{n} \times \mathbb{H}^{n} \times \mathbb{R}_{+} \rightarrow \mathbb{R}_{+}$is well defined (see [7] and references therein for its explicit construction and main properties). Then the unique bounded solution of the Cauchy problem

$$
\begin{cases}\frac{\partial u}{\partial t}=\Delta_{H} u & \text { in } \mathbb{H}^{n} \times \mathbb{R}_{+} \\ u=u_{0} & \text { in } \mathbb{H}^{n} \times\{0\}\end{cases}
$$

with Cauchy data $u_{0}$ bounded in $\mathbb{H}^{n}$ is

$$
u(x, t):=\int_{\mathbb{H}^{n}} G(x, y, t) u_{0}(y) d \mu_{y} \quad\left((x, t) \in \mathbb{H}^{n} \times \mathbb{R}_{+}\right),
$$

where $d \mu_{y}$ denotes the volume element of $\mathbb{H}^{n}$.

It is known [7] that there exists $c_{n}>0$ such that for any $x, y \in \mathbb{H}^{n}$ and $t \in \mathbb{R}_{+}$,

$$
\frac{1}{c_{n}} h_{n}(d(x, y), t) \leq G(x, y, t) \leq c_{n} h_{n}(d(x, y), t),
$$

where

$$
h_{n}(d, t):=(4 \pi t)^{-n / 2}(1+d)(1+d+t)^{(n-3) / 2} e^{-\lambda_{1} t-\frac{n-1}{2} d-\frac{d^{2}}{4 t}} \quad(d \geq 0, t>0) .
$$

Hence there exists $C_{n}>0$ such that for any $t \in \mathbb{R}_{+}$

$$
\sup _{x, y \in \mathbb{H}^{n}} G(x, y, t) \leq C_{n} \frac{(1+t)^{(n-3) / 2}}{t^{n / 2}} e^{-\lambda_{1} t} .
$$

(b) The hyperboloid model of $\mathbb{H}^{n}$, denoted by $\mathbb{I}^{n}$, is given by the hyperboloid

$$
\left\{\left(x_{1}, \ldots, x_{n}, x_{n+1}\right) \in \mathbb{R}^{n+1} \mid x_{1}^{2}+\cdots+x_{n}^{2}-x_{n+1}^{2}=-1, x_{n+1}>0\right\}
$$

with the metric induced on its tangent bundle by the Euclidean metric of $\mathbb{R}^{n+1}$.

For any $x \in \mathbb{I}^{n}$ and $y$ in the tangent space of $\mathbb{I}^{n}$ at $x$, the geodesic starting from $x$ with tangent vector $y$ is given by

$$
\gamma(t):=x \cosh t+y \sinh t \quad(t \in \mathbb{R}) .
$$


Observe that the map

$$
\phi: \mathbb{D}^{n} \rightarrow \mathbb{I}^{n}, \quad \phi(x):=\frac{\left(2 x, 1+|x|^{2}\right)}{1-|x|^{2}},
$$

is bijective and isometric. Then the Klein model of $\mathbb{H}^{n}$, denoted by $\mathbb{K}^{n}$, is defined using the bijective map

$$
\psi: \mathbb{R}^{n} \supseteq B_{1} \rightarrow \mathbb{I}^{n}, \quad \psi(x):=\frac{(x, 1)}{\sqrt{1-|x|^{2}}}
$$

(from a geometrical viewpoint, the point $\psi(x) \in \mathbb{R}^{n+1}$ for $x \in B_{1}$ is the intersection of $\mathbb{I}^{n}$ with the line passing through the point $(x, 1) \in \mathbb{R}^{n+1}$ and the origin in $\left.\mathbb{R}^{n+1}\right)$. By definition, the Klein model $\mathbb{K}^{n}$ is $B_{1}$ equipped with the metric obtained by transporting the hyperbolic metric of $\mathbb{I}^{n}$ along $\psi$.

Remark 2.1. Observe for further purposes that in $\mathbb{K}^{n}$ :

(i) geodesics are the traces of ordinary affine lines in $\mathbb{K}^{n}$ (see [12]);

(ii) for any convex subset $A$ of $\mathbb{K}^{n}$ and any point $x \in \mathbb{K}^{n} \backslash A$ there exists a hyperplane $\pi$ such that $x \in \pi$ and $\pi \cap A=\emptyset$;

(iii) for any convex subset $A$ of $\mathbb{K}^{n}$ and any entire geodesic $\gamma \subseteq \mathbb{K}^{n}$ which does not intersect $A$, there exists a hyperplane $\pi \subseteq \mathbb{K}^{n}$ such that $\gamma \subseteq \pi$ and $\pi \cap A=\emptyset$.

Furthermore, since the map $\phi^{-1} \circ \psi: \mathbb{K}^{n} \rightarrow \mathbb{D}^{n}$ is bijective and transforms geodesics of $\mathbb{K}^{n}$ onto those of $\mathbb{D}^{n}$, properties (ii)-(iii) hold true in $\mathbb{D}^{n}$, too.

(c) Finally, consider the Poincaré half-space model of the hyperbolic space, denoted by $\mathbb{U}^{n}$. This is the upper half-space $\left\{x \in \mathbb{R}^{n} \mid x_{n}>0\right\}$ endowed with the metric

$$
d s^{2}:=\frac{1}{x_{n}^{2}} \sum_{i=1}^{n} d x_{i}^{2}
$$

Observe that inversion in a sphere with radius $\sqrt{2}$ and center at the south pole of $\mathbb{D}^{n}$ maps $\mathbb{U}^{n}$ onto $\mathbb{D}^{n}$ (see [12]). Therefore $\mathbb{U}^{n}$ is isometric to $\mathbb{D}^{n}$, thus to $\mathbb{I}^{n}$.

For any $\bar{x} \in \partial \mathbb{U}^{n}=\left\{x \in \mathbb{R}^{n} \mid x_{n}=0\right\} \cup\{\infty\}$, horospheres with center at $\bar{x}$ are either hyperplanes of equation $x_{n}=\xi$ for some $\xi>0$, if $\bar{x}=\{\infty\}$, or spheres of $\mathbb{R}^{n}$ contained in $\mathbb{U}^{n}$ tangent in $\bar{x}$ to $\left\{x \in \mathbb{R}^{n} \mid x_{n}=0\right\}$, if $\bar{x}_{n}=0$ (see e.g. [5]). Clearly, for any $\bar{x} \in \partial \mathbb{U}^{n}$ with $\bar{x}_{n}=0$, a horosphere with center at $\bar{x}$ can be isometrically mapped into a horosphere with center at $\infty$.

For any $x=\left(x_{1}, \ldots, x_{n}\right), y=\left(y_{1}, \ldots, y_{n}\right) \in \mathbb{U}^{n}$ we have

$$
d(x, y)=\cosh ^{-1}\left[1+\frac{\left(x_{1}-y_{1}\right)^{2}+\cdots+\left(x_{n}-y_{n}\right)^{2}}{2 x_{n} y_{n}}\right] .
$$

The Laplace-Beltrami operator on $\mathbb{U}^{n}$ can be expressed as follows:

$$
\Delta_{H} u=x_{n}^{2} \Delta u+(2-n) x_{n} \frac{\partial u}{\partial x_{n}},
$$

where $\Delta$ denotes the Laplacian in $\mathbb{R}^{n}$. 


\section{Main results}

Let us first recall the following result concerning equation (1.8) (see [3, Theorem 4.1 and Lemma 4.3]).

Proposition 3.1. Let assumptions $\left(H_{0}\right)$ and either $\left(H_{1}\right)$ or $\left(H_{2}\right)$ be satisfied. Then there exists $c_{0}>0$ with the following properties:

(i) for $\kappa=c_{0}$ equation (1.8) admits a decreasing solution $q^{*}$ in $\mathbb{R}$ satisfying

$$
\lim _{\xi \rightarrow-\infty} q^{*}(\xi)=1, \quad \lim _{\xi \rightarrow \infty} q^{*}(\xi)=0
$$

(ii) for any $\kappa \in\left(0, c_{0}\right)$ there exists $\gamma_{\kappa} \in(0,1)$ such that: for any $\eta \in\left(\gamma_{\kappa}, 1\right)$ there exist $b=b(\kappa, \eta)>0$ and a solution q to equation (1.8) satisfying

$$
q(0)=\eta, \quad q^{\prime}(0)=0, \quad q(b)=0, \quad q^{\prime}<0 \quad \text { in }(0, b]
$$

(iii) for any $\kappa \in\left(c_{0}, \infty\right)$ there exists a solution $q$ to equation $(1.8)$ in $\mathbb{R}_{+}$such that

$$
q(0)=1, \quad q^{\prime}<0 \quad \text { in } \mathbb{R}_{+}, \quad q(\xi) \rightarrow 0 \quad \text { as } \xi \rightarrow \infty .
$$

\subsection{Extinction versus propagation}

If the function $f$ is of KPP type, the following holds.

Theorem 3.2. Let assumptions $\left(H_{0}\right),\left(H_{1}\right)$ and $\left(H_{1}^{\prime}\right)$ be satisfied. Let $u_{0} \not \equiv 0$, and let $u$ be the corresponding solution of problem (1.1).

(i) Suppose that $u_{0}$ has compact support and $c_{0}<n-1$. Then

$$
\lim _{t \rightarrow \infty} u(x, t)=0 \quad \text { uniformly in } \mathbb{H}^{n} .
$$

(ii) Suppose that $c_{0}>n-1$. Then

$$
\lim _{t \rightarrow \infty} u(x, t)=1 \quad \text { uniformly on compact subsets of } \mathbb{H}^{n} .
$$

Observe that a function $f$ of KPP type cannot satisfy condition (1.11). If (1.11) holds, we have the following result.

Theorem 3.3. Let assumptions $\left(H_{0}\right)$ and (1.11) be satisfied, and let $u_{0}$ be sufficiently small. Then equality (3.4) holds for the corresponding solution of problem (1.1).

Remark 3.1. By " $u_{0}$ sufficiently small" in the above statement we mean $u_{0}(x) \leq$ $w(\rho(x))$ for any $x \in \mathbb{H}^{n}$, the function $w=w(\rho)$ being defined in (4.19).

Results analogous to those of Theorem 3.2 hold if $f$ is of Allen-Cahn type and suitable assumptions on the size of $u_{0}$ are made. In fact, the following holds. 
Theorem 3.4. Let assumptions $\left(H_{0}\right)$ and $\left(H_{2}\right)$ be satisfied. Let $u$ be the solution of problem (1.1).

(i) If $\sup _{\mathbb{H}^{n}} u_{0}<a$, then equality (3.4) holds.

(ii) Suppose that $u_{0}$ is suitably large and $c_{0}>n-1$. Then equality (3.5) holds.

Remark 3.2. By " $u_{0}$ suitably large" in the above statement we mean $u_{0} \geq v_{0}$ in $\mathbb{H}^{n}$, the function $v_{0}$ being defined in (4.20).

The conclusion of Theorem 3.4(i) holds true if $\left(\mathrm{H}_{2}\right)$ is replaced by the weaker assumption:

$$
\left\{\begin{array}{l}
\text { there exists } a \in(0,1) \text { such that } f(u) \leq 0 \text { for any } u \in[0, a] \\
f(\bar{u})>0 \text { for some } \bar{u} \in(a, 1)
\end{array}\right.
$$

In fact, set

$$
\sigma \equiv \sigma(\eta):=\sup _{u \in(\eta, 1)} \frac{f(u)}{u-\eta}
$$

for any fixed $\eta \in[0, a)$. Then we have the following result, which is the counterpart of [3, Theorem 6.1].

Theorem 3.5. Let assumptions $\left(H_{0}\right)$ and $\left(H_{4}\right)$ be satisfied. Let $u$ be the solution to problem (1.1). Let $\eta \in[0, a)$ be fixed and either of the following assumptions be satisfied:

(i) $\sigma(\eta) \leq \lambda_{1}$;

(ii) $\lambda_{1}<\sigma(\eta)<\infty$ and there exists $t_{0}>0$ such that

$$
\int_{\mathbb{H}^{n}}\left[u_{0}(y)-\eta\right]_{+} d \mu_{y} \leq \frac{t_{0}^{n / 2}}{C_{n}\left(1+t_{0}\right)^{(n-3) / 2}} e^{\left[\lambda_{1}-\sigma(\eta)\right] t_{0}}(a-\eta),
$$

where $C_{n}>0$ is the constant in inequality (2.15).

Then

$$
\limsup _{t \rightarrow \infty} u(x, t) \leq \eta \quad \text { uniformly in } \mathbb{H}^{n} .
$$

Moreover, if $f(u)<0$ for any $u \in(0, \eta]$, then equality (3.4) holds.

\subsection{Speed of propagation and asymptotical symmetry}

Let us now address the case of propagation. Concerning the spreading speed, we have the following result.

Theorem 3.6. Let assumptions $\left(H_{0}\right)$, and either $\left(H_{1}\right)-\left(H_{1}^{\prime}\right)$ or $\left(H_{2}\right)$ be satisfied. Let $u_{0} \not \equiv 0$ have compact support, and let $u_{0}$ be suitably large if $\left(\mathrm{H}_{2}\right)$ holds. Moreover, assume $c_{0}>n-1$.

(i) Let $c>c_{0}-(n-1)$. Then for every $y \in \mathbb{H}^{n}$,

$$
\lim _{t \rightarrow \infty} \sup _{d(x, y)>c t} u(x, t)=0 .
$$

(ii) Let $0<c<c_{0}-(n-1)$. Then for every $y \in \mathbb{H}^{n}$,

$$
\lim _{t \rightarrow \infty} \inf _{d(x, y)<c t} u(x, t)=1 .
$$


Let us also prove some geometrical properties of the level sets of the solution of problem (1.1), which are the counterpart of [13, Theorem] in $\mathbb{R}^{n}$. This is the content of the following

Theorem 3.7. Let the assumptions of Theorem 3.6 be satisfied. Then for any a $\in(0,1)$ and $t \in \mathbb{R}_{+}$sufficiently large the following holds:

(i) the level set

$$
\Gamma_{a}(u ; t):=\left\{x \in \mathbb{H}^{n} \mid u(x, t)=a\right\} \quad\left(t \in \mathbb{R}_{+}\right)
$$

is a smooth $(n-1)$-dimensional submanifold of $\mathbb{H}^{n}$;

(ii) every geodesic orthogonal to $\Gamma_{a}(u ; t)$ intersects the convex hull of the support of $u_{0}$.

Since the size of $\Gamma_{a}(u ; t)$ tends to infinity as $t \rightarrow \infty$, while that of supp $u_{0}$ remains unchanged, the above theorem implies, roughly, that the shape of the expanding front $\Gamma_{a}(u ; t)$ converges to that of a sphere, at least in the $C^{1}$ sense. Furthermore, one can derive from the above theorem the following corollary.

Corollary 3.8. Let the assumptions of Theorem 3.6 be satisfied. Fix a point $P \in \operatorname{supp} u_{0}$, and let $\rho_{\min }(t)$ (respectively $\rho_{\max }(t)$ ) denote the minimal (respectively maximal) geodesic distance from $P$ to $\Gamma_{a}(u ; t)$. Then there exists a constant $M>0$ such that

$$
\rho_{\max }(t)-\rho_{\min }(t) \leq M \quad \text { for all } t \geq 0 .
$$

\subsection{Horospheric waves}

Let us first state the following definition.

Definition 3.1. By a horospheric wave solution of problem (1.1) we mean any solution whose level sets $\Gamma_{a}(u ; t)\left(a \in(0,1), t \in \mathbb{R}_{+}\right)$are horospheres of the form (1.17):

$$
\Gamma_{a}(u ; t)=\left\{x \in \mathbb{U}^{n} \mid x_{n}=K_{a} e^{-\kappa t}\right\} \quad\left(K_{a}>0, \kappa \in \mathbb{R}\right)
$$

in the Poincaré half-space model $\mathbb{U}^{n}$.

Concerning existence of horospheric waves, the following result will be proven.

Theorem 3.9. Let assumptions $\left(H_{0}\right)$ and either $\left(H_{1}\right)$ or $\left(H_{2}\right)$ be satisfied. Suppose $c_{0}>$ $n-1$. Then there exists a horospheric wave which in the Poincaré half-space model $\mathbb{U}^{n}$ takes the form (1.18):

$$
u\left(x_{1}, \ldots, x_{n}, t\right):=q^{*}\left(-\log x_{n}-c^{*} t\right) \quad\left(\left(x_{1}, \ldots, x_{n}, t\right) \in \mathbb{U}^{n} \times \mathbb{R}_{+}\right) .
$$

Observe that the existence of the profile function $q^{*}$ is ensured by Proposition 3.1(i). The quantity $c^{*}:=c_{0}-(n-1)$ is called the speed of the horospheric wave.

Consider problem (1.1) in the half-space model $\mathbb{U}^{n}$, namely

$$
\begin{cases}\frac{\partial u}{\partial t}=\Delta_{H} u+f(u) & \text { in } \mathbb{U}^{n} \times \mathbb{R}_{+}, \\ u=u_{0} & \text { in } \mathbb{U}^{n} \times\{0\} .\end{cases}
$$


Remarkably, horospheric waves provide upper and lower bounds for large times of any solution of problem (3.12), as plane waves in $\mathbb{R}^{n}$ do for solutions of problem (1.3). To address this point, let us think of the function $f$ as defined on $\mathbb{R}$ and satisfying the following assumption (which implies both $\left(H_{0}\right)$ and $\left(H_{2}\right)$ ):

(i) $f \in C^{1}(\mathbb{R}), f(0)=f(1)=0, f^{\prime}(0)<0, f^{\prime}(1)<0, \int_{0}^{1} f(u) d u>0$;

(ii) there exists $a \in(0,1)$ such that $f(u)>0$ if $u \in(-\infty, 0) \cup(a, 1)$, and $f(u)<0$ if $u \in(0, a) \cup(1, \infty)$.

Then the following result can be proven.

Theorem 3.10. Let assumption $\left(H_{5}\right)$ be satisfied, and let $u$ be any solution of problem (3.12). Suppose that

$$
\limsup _{x_{n} \rightarrow 0^{+}} \sup _{x^{\prime} \in \mathbb{R}^{n-1}} u_{0}\left(x^{\prime}, x_{n}\right)<a, \quad \liminf _{x_{n} \rightarrow \infty} \inf _{x^{\prime} \in \mathbb{R}^{n-1}} u_{0}\left(x^{\prime}, x_{n}\right)>a .
$$

Then there exist $k_{*}, k^{*} \in \mathbb{R}_{+}$such that

$$
q^{*}\left(-\log \left(k_{*} x_{n}\right)-c^{*} t\right) \leq u\left(x^{\prime}, x_{n}, t\right) \leq q^{*}\left(-\log \left(k^{*} x_{n}\right)-c^{*} t\right)
$$

for every $\left(x^{\prime}, x_{n}\right) \in \mathbb{U}^{n}$ and $t \in \mathbb{R}_{+}$.

Let us add some remarks suggested by Theorem 3.10. For each given direction $\theta \in S^{n-1}$ in the Poincare disk model we can choose the direction of the $x_{n}$-axis along this $\theta$ direction, and express the solution in the half-space model $\mathbb{U}^{n}$ with respect to this choice of the $x_{n}$-axis. By this choice, it is possible to define properly " the $\omega$-limit set in the direction $\theta$ " of any solution $u$ of problem (3.12).

By assumptions $(A)$ and $\left(H_{5}\right)(i)$, using standard comparison and compactness results it is easily seen that, for any $\theta \in S^{n-1}$, the $\omega$-limit set of $u$ in the direction $\theta$ is nonempty (see e.g. [16] for details). If $\tilde{u}=\tilde{u}\left(x^{\prime}, x_{n}, t\right)$ is any point of this set, it follows from inequality (3.14) that

$q^{*}\left(-\log \left(k_{*} x_{n}\right)-c^{*} t\right) \leq \tilde{u}\left(x^{\prime}, x_{n}, t\right) \leq q^{*}\left(-\log \left(k^{*} x_{n}\right)-c^{*} t\right) \quad\left(\left(x^{\prime}, x_{n}\right) \in \mathbb{U}^{n}, t \in \mathbb{R}_{+}\right)$.

However, it is an open problem whether every $\omega$-limit point $\tilde{u}$ itself is a horospheric wave (see [6, Theorem 3.1] for the analogous result concerning plane waves in $\mathbb{R}^{n}$ ).

\section{Extinction versus propagation: proofs}

Let us first prove Theorem 3.2.

Proof of Theorem 3.2. (i) By assumption, there exists $R>0$ such that $\operatorname{supp} u_{0} \subseteq \mathfrak{B}_{R}$. Hence there exists a smooth function $\tilde{u}_{0}: \overline{\mathbb{R}}_{+} \rightarrow[0,1], \tilde{u}_{0}=\tilde{u}_{0}(\rho), \tilde{u}_{0}^{\prime} \leq 0$ in $\overline{\mathbb{R}}_{+}$, $\tilde{u}_{0}(\rho)=0$ for any $\rho \geq R$, such that

$$
u_{0}(x) \leq \tilde{u}_{0}(\rho(x)) \quad \text { for any } x \in \mathbb{H}^{n} .
$$


Let $\tilde{u}$ be the solution of the problem

$$
\begin{cases}\frac{\partial u}{\partial t}=\frac{\partial^{2} u}{\partial \rho^{2}}+(n-1) \operatorname{coth} \rho \frac{\partial u}{\partial \rho}+f(u) & \text { in } \mathbb{R}_{+} \times \mathbb{R}_{+} \\ \frac{\partial u}{\partial \rho}=0 & \text { in }\{0\} \times \mathbb{R}_{+} \\ u=\tilde{u}_{0} & \text { in } \mathbb{R}_{+} \times\{0\} .\end{cases}
$$

Then $\tilde{u}(\rho(x), t)$ solves problem (1.1) with Cauchy data $\tilde{u}_{0}(\rho(x))$ (see (2.12)). By (4.1) and comparison results (see [17]) we obtain

$$
0 \leq u(x, t) \leq \tilde{u}(\rho(x), t) \quad \text { for any }(x, t) \in \mathbb{H}^{n} \times \mathbb{R}_{+} .
$$

On the other hand, consider the problem

$$
\begin{cases}\frac{\partial w}{\partial t}=\frac{\partial^{2} w}{\partial \rho^{2}}+(n-1) \frac{\partial w}{\partial \rho}+f(w) & \text { in } \mathbb{R}_{+} \times \mathbb{R}_{+} \\ \frac{\partial w}{\partial \rho}=0 & \text { in }\{0\} \times \mathbb{R}_{+} \\ w=\tilde{u}_{0} & \text { in } \mathbb{R}_{+} \times\{0\} .\end{cases}
$$

We shall prove the following

Claim 1. Let $c_{0}<n-1$. Then the solution $w$ of problem (4.4) satisfies

$$
\begin{aligned}
& \sup _{\rho \in \mathbb{R}_{+}} w(\rho, t) \rightarrow 0 \quad \text { as } t \rightarrow \infty, \\
& \frac{\partial w}{\partial \rho} \leq 0 \quad \text { in } \mathbb{R}_{+} \times \mathbb{R}_{+} .
\end{aligned}
$$

From the above claim the conclusion easily follows. In fact, since coth $\rho \geq 1$ and $\partial w / \partial \rho$ $\leq 0, w$ is a supersolution of problem (4.2). Therefore, by comparison results

$$
0 \leq \tilde{u}(\rho, t) \leq w(\rho, t) \quad \text { in } \mathbb{R}_{+} \times \mathbb{R}_{+},
$$

whence by (4.3) and (4.5),

$$
0 \leq \sup _{x \in \mathbb{H}^{n}} u(x, t) \leq \sup _{\rho \in \mathbb{R}_{+}} \tilde{u}(\rho, t) \rightarrow 0 \quad \text { as } t \rightarrow \infty .
$$

It remains to prove Claim 1 . To this end observe that, since by assumption $c_{0}<n-1$, by Proposition 3.1(iii) the ordinary differential equation

$$
q^{\prime \prime}+(n-1) q^{\prime}+f(q)=0
$$

has a solution $q=q(\xi)$ in $\mathbb{R}_{+}$such that

$$
q(0)=1, \quad q^{\prime}<0 \quad \text { in } \mathbb{R}_{+}, \quad q(\xi) \rightarrow 0 \quad \text { as } \xi \rightarrow \infty .
$$


Set

$$
\varphi(\xi):= \begin{cases}1 & \text { if } \xi<R \\ q(\xi-R) & \text { if } \xi \geq R .\end{cases}
$$

Let $v$ be the solution of the problem

$$
\begin{cases}\frac{\partial v}{\partial t}=\frac{\partial^{2} v}{\partial \xi^{2}}+(n-1) \frac{\partial v}{\partial \xi}+f(v) & \text { in } \mathbb{R} \times \mathbb{R}_{+}, \\ v=\varphi & \text { in } \mathbb{R} \times\{0\} .\end{cases}
$$

The proof of [3, Theorem 5.1] shows that

$$
\frac{\partial v}{\partial \xi} \leq 0 \quad \text { in } \mathbb{R} \times \mathbb{R}_{+} \quad \text { and } \quad \lim _{t \rightarrow \infty} v(\xi, t)=0
$$

for any $\xi \in \mathbb{R}$. Since $\tilde{u}_{0} \leq \varphi$ in $\mathbb{R}_{+}$, by the inequality in (4.9) the function $v$ is a supersolution of problem (4.4). Hence $w \leq v$ in $\mathbb{R}_{+} \times \mathbb{R}_{+}$. This fact and (4.9) yield (4.5).

To prove (4.6), consider the problem

$$
\begin{cases}\frac{\partial z}{\partial t}=\frac{\partial^{2} z}{\partial \rho^{2}}+(n-1) \frac{\partial z}{\partial \rho}+f^{\prime}(w) z & \text { in } \mathbb{R}_{+} \times \mathbb{R}_{+} \\ z=0 & \text { in }\{0\} \times \mathbb{R}_{+} \\ z=0 & \text { in } \mathbb{R}_{+} \times\{0\}\end{cases}
$$

where $w$ is the solution of problem (4.4). Since by assumption $\tilde{u}_{0}^{\prime} \leq 0$, the function $\partial w / \partial \rho$ is a subsolution of the above problem, whereas $z \equiv 0$ is a solution. Hence by comparison (4.6) follows. This completes the proof of Claim 1, thus of (i).

(ii) Under the present assumptions inequality (1.15) holds, $\lambda_{1}$ denoting the infimum of the $L^{2}$-spectrum of the operator $-\Delta_{H}$ in $\mathbb{H}^{n}$. Consider the eigenvalue problem

$$
\begin{cases}\Delta_{H} \varphi+\left[f^{\prime}(0)+\mu\right] \varphi=0 & \text { in } \mathfrak{B}_{R} \\ \varphi=0 & \text { on } \partial \mathfrak{B}_{R}(R>0) .\end{cases}
$$

Denote by $\mu_{1}=\mu_{1}\left(\mathfrak{B}_{R}\right)$ the first eigenvalue of (4.10) and by $\varphi_{1}=\varphi_{1}(\rho)>0$ the corresponding eigenfunction. Clearly,

$$
\mu_{1}\left(\mathfrak{B}_{R}\right)=\lambda_{1}\left(\mathfrak{B}_{R}\right)-f^{\prime}(0)
$$

(see (2.10)), where $\lambda_{1}\left(\mathfrak{B}_{r}\right)$ denotes the first eigenvalue of $-\Delta_{H}$ in $\mathfrak{B}_{r}$ with Dirichlet zero boundary conditions. Hence by (1.15) and (2.9) there exists $R_{0}>0$ such that $\mu_{1}\left(\mathfrak{B}_{R}\right)<0$ for any $R>R_{0}$.

Set

$$
w_{\varepsilon}(\rho):= \begin{cases}\varepsilon \varphi_{1}(\rho) & \text { in } \mathfrak{B}_{R}, \\ 0 & \text { otherwise }\end{cases}
$$

with $R>R_{0}$ fixed. Since $\mu_{1}\left(\mathfrak{B}_{R}\right)<0$, it is easily seen that there exists $\varepsilon_{0}>0$ such that for any $\varepsilon \in\left(0, \varepsilon_{0}\right)$ the function $w_{\varepsilon}$ is a subsolution of the equation

$$
\Delta_{H} v+f(v)=0 \quad \text { in } \mathbb{H}^{n} .
$$


On the other hand, every solution $u=u(x, t)$ of problem (1.1) with Cauchy data $u_{0} \not \equiv 0$ satisfies $u(\cdot, \bar{t})>0$ in $\mathbb{H}^{n}$ for any $\bar{t} \in \mathbb{R}_{+}$(this is a standard consequence of the strong maximum principle; see the form (2.11) of the operator $\Delta_{H}$ in $\left.\mathbb{D}^{n}\right)$. Choose $\varepsilon \in\left(0, \varepsilon_{0}\right)$ so small that

$$
w_{\varepsilon}(\rho(x)) \leq u(x, \bar{t}) \quad \text { for any } x \in \mathbb{H}^{n} .
$$

Then by comparison arguments

$$
u_{\varepsilon}(\cdot, t) \leq u(\cdot, t+\bar{t}) \leq 1 \quad \text { in } \mathbb{H}^{n}
$$

for any $t \in \mathbb{R}_{+}$, where $u_{\varepsilon}$ denotes the solution of the problem

$$
\begin{cases}\frac{\partial u}{\partial t}=\Delta_{H} u+f(u) & \text { in } \mathbb{H}^{n} \times \mathbb{R}_{+}, \\ u=w_{\varepsilon} & \text { in } \mathbb{H}^{n} \times\{0\} .\end{cases}
$$

By standard arguments,

(a) the function $t \mapsto u_{\varepsilon}(x, t)$ is nondecreasing in $\mathbb{R}_{+}$for any $x \in \mathbb{H}^{n}$;

(b) its pointwise limit

$$
u_{\infty}(x):=\lim _{t \rightarrow \infty} u_{\varepsilon}(x, t) \quad\left(x \in \mathbb{H}^{n}\right)
$$

is a solution of equation (4.12);

(c) the convergence $u_{\varepsilon}(\cdot, t) \rightarrow u_{\infty}$ as $t \rightarrow \infty$ is uniform on compact subsets of $\mathbb{H}^{n}$.

Therefore the conclusion will follow from inequality (4.13) if we prove that

$$
u_{\infty} \equiv 1 \quad \text { in } \mathbb{H}^{n} \text {. }
$$

To this end, choose $\varepsilon \in\left(0, \varepsilon_{0}\right)$ so small that

$$
0 \leq w_{\varepsilon}(x)<u_{\infty}(x) \quad \text { for any } x \in \mathbb{H}^{n} .
$$

We shall prove the following

Claim 2. We have

$$
B:=\left\{y \in \mathbb{H}^{n} \mid w_{\varepsilon}\left(\tau_{y}(x)\right)<u_{\infty}(x) \text { for any } x \in \mathbb{H}^{n}\right\}=\mathbb{H}^{n},
$$

where $\tau_{y}: \mathbb{H}^{n} \rightarrow \mathbb{H}^{n}$ is the continuous family of isometries defined in (2.6).

From the above claim equality (4.15) follows easily. In fact, since $B=\mathbb{H}^{n}$, we obtain in particular

This implies

$$
w_{\varepsilon}\left(\tau_{x}(x)\right)=w_{\varepsilon}(0)<u_{\infty}(x) \quad \text { for any } x \in \mathbb{H}^{n} .
$$

$$
\xi(t) \leq u_{\infty}(x) \quad \text { for any } x \in \mathbb{H}^{n}, t \in \mathbb{R}_{+},
$$

where $\xi$ is the solution of the problem

$$
\xi^{\prime}=f(\xi), \quad \xi(0)=w_{\varepsilon}(0) .
$$

Since $\xi(t) \rightarrow 1$ as $t \rightarrow \infty$, inequality (4.17) implies (4.15).

To prove Claim 2, observe first that $0 \in B$. In fact, by (4.16),

$$
w_{\varepsilon}\left(\tau_{0}(x)\right)=w_{\varepsilon}(x)<u_{\infty}(x) \quad \text { for any } x \in \mathbb{H}^{n} .
$$


By the continuity of the map $y \mapsto \tau_{y}(x)$ for any fixed $x \in \mathbb{H}^{n}$ (see (2.6)), the set $B$ is open. Moreover, $B$ is also closed. In fact, let $\left\{y_{n}\right\} \subseteq B$ and $y \in \mathbb{H}^{n}$ be such that $d\left(y_{n}, y\right) \rightarrow 0$. Then by the continuity of $w_{\varepsilon}$

$$
w_{\varepsilon}\left(\tau_{y}(x)\right) \leq u_{\infty}(x) \text { for any } x \in \mathbb{H}^{n} .
$$

However, if we had

$$
w_{\varepsilon}\left(\tau_{y}(\bar{x})\right)=u_{\infty}(\bar{x}) \quad \text { for some } \bar{x} \in \mathbb{H}^{n},
$$

we would have a contradiction with the strong maximum principle, since $u_{\infty}$ a solution of equation (4.12) and $w_{\varepsilon} \circ \tau_{y}$ a subsolution of the same equation (for $w_{\varepsilon}$ is a subsolution and $\Delta_{H}$ commutes with isometries of $\mathbb{H}^{n}$ ). Hence the inequality in (4.18) is strict, which proves that $y \in B$, thus $B$ is closed.

Since the set $B$ is open, closed and nonempty, Claim 2 follows. This completes the proof of claim (ii), thus the conclusion follows.

Remark 4.1. The arguments used in the proof of Theorem 3.2(ii) show that $v \equiv 1$ is the unique nontrivial solution of equation (4.12) such that $0 \leq v \leq 1$, if (1.15) holds.

Let us recall the following result (see [4, Lemma A.1]).

Lemma 4.1. For any $k>0$ there exists a unique radial ground state $\phi=\phi(\rho)$ of equation (1.16) such that $\phi(0)=k$. Moreover, $\lim _{\rho \rightarrow \infty} \phi(\rho)=0$.

Proof of Theorem 3.3. By assumption (1.11) there exist $L>0$ and $\sigma \in(0,1)$ such that $f(u) \leq L u^{p}$ for any $u \in(0, \sigma)$. Set

$$
w:=c \phi \quad \text { in } \mathbb{H}^{n},
$$

where $\phi$ is the ground state mentioned in Lemma 4.1 and

$$
0<c<\frac{1}{\|\phi\|_{\infty}} \min \left\{\sigma,\left(\frac{\lambda_{1}}{L}\right)^{1 /(p-1)}\right\} .
$$

Define also

$$
\bar{u}(x, t):=e^{-\lambda_{1} t} \zeta(t) w(\rho(x)) \quad \text { for any }(x, t) \in \mathbb{H}^{n} \times \overline{\mathbb{R}}_{+},
$$

where

$$
\zeta(t):=\left\{1-\frac{L}{\lambda_{1}}\|w\|_{\infty}^{p-1}\left[1-e^{-(p-1) \lambda_{1} t}\right]\right\}^{-1 /(p-1)} \quad\left(t \in \overline{\mathbb{R}}_{+}\right) .
$$

Assuming that $u_{0}(x) \leq w(\rho(x))$ for any $x \in \mathbb{H}^{n}$, it is easily seen that $\bar{u}$ is a supersolution to problem (1.1). In fact,

(a) for any $(x, t) \in \mathbb{H}^{n} \times \overline{\mathbb{R}}_{+}$,

$$
\bar{u}(x, t) \leq w(\rho(x)) \leq c\|\phi\|_{\infty}<\sigma,
$$

hence $f(\bar{u}) \leq L \bar{u}^{p}$ in $\mathbb{H}^{n} \times \overline{\mathbb{R}}_{+}$; 
(b) by (a) and the definition of $\bar{u}$,

$$
\frac{\partial \bar{u}}{\partial t}-\Delta_{H} \bar{u}-f(\bar{u}) \geq \frac{\partial \bar{u}}{\partial t}-\Delta_{H} \bar{u}-L \bar{u}^{p} \geq e^{-\lambda_{1} t} w\left[\zeta^{\prime}-L\|w\|_{\infty}^{p-1} e^{-(p-1) \lambda_{1} t} \zeta^{p}\right]=0 ;
$$

(c) by assumption, $u_{0}(x) \leq w(\rho(x))=\bar{u}(x, 0)$.

Then by comparison results we have $0 \leq u \leq \bar{u}$ in $\mathbb{H}^{n} \times \overline{\mathbb{R}}_{+}$. Since

$$
\sup _{\mathbb{H}^{n}} \bar{u} \leq\|w\|_{\infty} e^{-\lambda_{1} t} \rightarrow 0 \quad \text { as } t \rightarrow \infty,
$$

the conclusion follows.

Let us now prove Theorem 3.4. To prove claim (ii) of that theorem a preliminary result is needed.

Let $c_{0}>n-1$. By Proposition 3.1(ii), for any $c \in\left(0, c_{0}-(n-1)\right)$ there exists $\gamma_{c} \in(0,1)$ such that: for any $\eta \in\left(\gamma_{c}, 1\right)$ there exist $b=b(c, \eta)>0$ and a solution $q$ of the equation

$$
q^{\prime \prime}+(c+n-1) q^{\prime}+f(q)=0
$$

such that

$$
q(0)=\eta, \quad q^{\prime}(0)=0, \quad q(b)=0, \quad q^{\prime}<0 \quad \text { in }(0, b] .
$$

For any fixed $R>0$ define

$$
v_{0}(\rho):= \begin{cases}\eta & \text { if } \rho \leq R, \\ q(\rho-R) & \text { if } R<\rho \leq R+b, \\ 0 & \text { if } R+b<\rho .\end{cases}
$$

Concerning the solution of the parabolic problem

$$
\begin{cases}\frac{\partial v}{\partial t}=\Delta_{H} v+f(v) & \text { in } \mathbb{H}^{n} \times \mathbb{R}_{+}, \\ v=v_{0}(\rho) & \text { in } \mathbb{H}^{n} \times\{0\},\end{cases}
$$

the following holds (see [3, Lemma 5.1]).

Lemma 4.2. Let assumptions $\left(H_{0}\right)$ and either $\left(H_{1}\right)$ or $\left(H_{2}\right)$ be satisfied. Let $c_{0}>n-1$, $c \in\left(0, c_{0}-(n-1)\right), \gamma_{c} \in(0,1)$ and $\eta \in\left(\gamma_{c}, 1\right)$ as above. Then for any $R>0$ such that

$$
\operatorname{coth} R<1+\frac{c}{n-1}
$$

the solution $v$ of problem (4.21) satisfies the inequality

$$
v(x, t) \geq \eta \quad \text { for any }(x, t) \in \mathbb{H}^{n} \times \mathbb{R}_{+} \text {such that } \rho(x) \leq R+k t,
$$

where

$$
k \equiv k(c):=c+(n-1)(1-\operatorname{coth} R)>0 .
$$

Therefore,

$$
\lim _{t \rightarrow \infty} v(x, t)=1 \quad \text { uniformly in compact subsets of } \mathbb{H}^{n} .
$$


Proof. Following [3], define

$$
W(x, t):=v_{0}(\rho(x)-k t) \quad\left((x, t) \in \mathbb{H}^{n} \times \mathbb{R}_{+}\right)
$$

with $k$ given by (4.24). It is easily seen that

$$
\frac{\partial W}{\partial t}-\Delta_{H} W-f(W)= \begin{cases}-f(\eta) & \text { if } \rho(x) \leq R+k t \\ q^{\prime}(\rho(x)-k t-R)[c-k+(n-1)(1-\operatorname{coth} \rho(x))] \\ 0 & \text { if } R+k t<\rho(x) \leq R+b+k t \\ 0 & \text { if } \rho(x)>R+b+k t .\end{cases}
$$

Observe that

$$
\begin{gathered}
q^{\prime}(\rho(x)-k t-R)<0, \\
c-k+(n-1)(1-\operatorname{coth} \rho(x)) \geq c-k+(n-1)(1-\operatorname{coth} R)=0
\end{gathered}
$$

if $R+k t<\rho(x) \leq R+b+k t$. Moreover, under the present assumptions, $f(\eta)>0$ if $\eta$ is sufficiently close to 1 .

Arguing as in [3], we find that $v \geq W$ in $\mathbb{H}^{n} \times \mathbb{R}_{+}$. Since by definition $W(x, t)=\eta$ if $\rho(x) \leq R+k t$, inequality (4.23) follows. Moreover, since $\eta$ is arbitrarily close to 1 , equality (4.25) follows from (4.23).

Proof of Theorem 3.4. (i) Let $\zeta=\zeta(t)$ be the solution of the problem

$$
\zeta^{\prime}=f(\zeta) \quad \text { in } \mathbb{R}_{+}, \quad \zeta(0)=\sup _{\mathbb{H}^{n}} u_{0} .
$$

Clearly, $\zeta$ is a supersolution of problem (1.1), thus by comparison results $0 \leq u(x, t)$ $\leq \zeta(t)$ for any $(x, t) \in \mathbb{H}^{n} \times \mathbb{R}_{+}$. Since by assumption $\zeta(0)<a$ and $\left(H_{2}\right)(\mathrm{i})$ holds, we have $\zeta(t) \rightarrow 0$ as $t \rightarrow \infty$. Hence claim (i) follows.

(ii) Let $u_{0}(\rho) \geq v_{0}(\rho)$ in $\mathbb{H}^{n}$, where $v_{0}$ is the function defined in (4.20). By comparison results we have $u \geq v$ in $\mathbb{H}^{n} \times \mathbb{R}_{+}, v$ being the solution of problem (4.21). Then by Lemma 4.2 the conclusion follows.

Proof of Theorem 3.5. Let $w$ be the solution of the problem

$$
\begin{cases}\frac{\partial w}{\partial t}=\Delta_{H} w+\sigma(\eta) w & \text { in } \mathbb{H}^{n} \times \mathbb{R}_{+}, \\ w=\left(u_{0}-\eta\right)_{+} & \text {in } \mathbb{H}^{n} \times\{0\} .\end{cases}
$$

Define

$$
v(x, t):=w(x, t)+\eta \quad\left((x, t) \in \mathbb{H}^{n} \times \mathbb{R}_{+}\right) .
$$

Since $w \geq 0$, it follows that $v \geq \eta$, whence by the definition of $\sigma(\eta)$,

$$
f(v) \leq \sigma(\eta)(v-\eta)=\sigma(\eta) w \quad \text { in } \mathbb{H}^{n} \times \mathbb{R}_{+} .
$$

Then

$$
\frac{\partial v}{\partial t}-\Delta_{H} v-f(v) \geq \frac{\partial w}{\partial t}-\Delta_{H} w-\sigma(\eta) w=0 \quad \text { in } \mathbb{H}^{n} \times \mathbb{R}_{+}
$$


Moreover,

$$
u_{0}(x) \leq v(x, 0) \quad \text { for any } x \in \mathbb{H}^{n} .
$$

Then by comparison results

$$
u(x, t) \leq v(x, t) \quad \text { for any }(x, t) \in \mathbb{H}^{n} \times \mathbb{R}_{+} .
$$

On the other hand, from (4.26), (4.27) and the estimate (2.15) we obtain

$$
\begin{aligned}
v(x, t) & =\eta+e^{\sigma(\eta) t} \int_{\mathbb{H}^{n}} G(x, y, t)\left[u_{0}(y)-\eta\right]_{+} d \mu_{y} \\
& \leq \eta+C_{n} \frac{(1+t)^{(n-3) / 2}}{t^{n / 2}} e^{\left[\sigma(\eta)-\lambda_{1}\right] t} \int_{\mathbb{H}^{n}}\left[u_{0}(y)-\eta\right]_{+} d \mu_{y}
\end{aligned}
$$

for any $(x, t) \in \mathbb{H}^{n} \times \mathbb{R}_{+}$. Inequality (4.29) and either assumption (i), (ii) imply that there exists $t_{0}>0$ such that $v\left(x, t_{0}\right) \leq a$, thus by inequality (4.28) we obtain $u\left(x, t_{0}\right) \leq a$ for any $x \in \mathbb{H}^{n}$. Since by assumption $f(a) \leq 0$, by comparison we obtain $u \leq a$ in $\mathbb{H}^{n} \times\left[t_{0}, \infty\right)$, thus $f(u) \leq 0$ in $\mathbb{H}^{n} \times\left[t_{0}, \infty\right)$ by assumption $\left(H_{4}\right)$.

Now let $z$ solve the problem

$$
\begin{cases}\frac{\partial z}{\partial t}=\Delta_{H} z & \text { in } \mathbb{H}^{n} \times\left(t_{0}, \infty\right), \\ z=v & \text { in } \mathbb{H}^{n} \times\left\{t_{0}\right\} .\end{cases}
$$

Since $f(u) \leq 0$ in $\mathbb{H}^{n} \times\left[t_{0}, \infty\right)$ and inequality (4.28) holds, by comparison results we have

$$
u(x, t) \leq z(x, t) \quad \text { in } \mathbb{H}^{n} \times\left[t_{0}, \infty\right) .
$$

On the other hand,

$$
\begin{aligned}
z(x, t) & =\int_{\mathbb{H}^{n}} G\left(x, y, t-t_{0}\right) v\left(y, t_{0}\right) d \mu_{y}=\eta+\int_{\mathbb{H}^{n}} G\left(x, y, t-t_{0}\right) w\left(y, t_{0}\right) d \mu_{y} \\
& =\eta+e^{\sigma(\eta) t_{0}} \int_{\mathbb{H}^{n}} G(x, y, t)\left[u_{0}(y)-\eta\right]_{+} d \mu_{y} \\
& \leq \eta+C_{n} e^{\sigma(\eta) t_{0}} \frac{(1+t)^{(n-3) / 2}}{t^{n / 2}} e^{-\lambda_{1} t} \int_{\mathbb{H}^{n}}\left[u_{0}(y)-\eta\right]_{+} d \mu_{y}
\end{aligned}
$$

for any $t \geq t_{0}$ (here use of (2.15) and (3.7) has been made). From inequalities (4.30)(4.31) and either assumption (i), (ii) we plainly obtain (3.8).

To conclude the proof, let $f(u)<0$ in $(0, \eta]$. Then by the continuity of $f$ there exists $\varepsilon \in(0, a-\eta)$ such that $f(u)<0$ for any $u \in(0, \eta+\varepsilon]$. On the other hand, by (4.30)-(4.31) there exists $t_{\varepsilon}>0$ such that

$$
u(x, t) \leq z(x, t)<\eta+\varepsilon / 2 \quad \text { for any }(x, t) \in \mathbb{H}^{n} \times\left[t_{\varepsilon}, \infty\right) .
$$

Let $\zeta$ solve the problem

$$
\zeta^{\prime}=f(\zeta) \quad \text { in }\left(t_{\varepsilon}, \infty\right), \quad \zeta\left(t_{\varepsilon}\right)=\eta+\varepsilon / 2
$$


Then by (4.32), $\zeta$ is a supersolution, whereas $u$ is a subsolution of the problem

$$
\begin{cases}\frac{\partial y}{\partial t}=\Delta_{H} y+f(y) & \text { in } \mathbb{H}^{n} \times\left(t_{\varepsilon}, \infty\right), \\ y=z & \text { in } \mathbb{H}^{n} \times\left\{t_{\varepsilon}\right\} .\end{cases}
$$

Hence by comparison results

$$
0 \leq u(x, t) \leq \zeta(t) \quad \text { for any }(x, t) \in \mathbb{H}^{n} \times\left[t_{\varepsilon}, \infty\right) .
$$

Since $\zeta(t) \rightarrow 0$ as $t \rightarrow \infty$, letting $t \rightarrow \infty$ in the above inequality we obtain (3.4).

\section{Speed of propagation and asymptotical symmetry: proofs}

Proof of Theorem 3.6. (i) Choose $\tilde{u}_{0}$ as in the proof of Theorem 3.2, and let $\tilde{u}$ be the corresponding solution of (4.2). Then inequality (4.3) holds.

Since by assumption $c+n-1>c_{0}$, there exists a solution $q=q(\xi)$ of the ordinary differential equation

$$
q^{\prime \prime}+(c+n-1) q^{\prime}+f(q)=0
$$

in $\mathbb{R}_{+}$such that

$$
q(0)=1, \quad q^{\prime}<0 \quad \text { in } \mathbb{R}_{+}, \quad q(\xi) \rightarrow 0 \quad \text { as } \xi \rightarrow \infty
$$

(see Proposition 3.1(iii)). Let $\varphi=\varphi(\xi)$ be the function defined in (4.7), so that $\tilde{u}_{0} \leq \varphi$ in $\mathbb{R}_{+}$, and let $v$ be the solution of the problem

$$
\begin{cases}\frac{\partial v}{\partial t}=\frac{\partial^{2} v}{\partial \xi^{2}}+(c+n-1) \frac{\partial v}{\partial \xi}+f(v) & \text { in } \mathbb{R} \times \mathbb{R}_{+}, \\ v=\varphi & \text { in } \mathbb{R} \times\{0\} .\end{cases}
$$

As in the proof of Theorem 3.2, the function $v$ has the properties (4.9), namely

$$
\frac{\partial v}{\partial \xi} \leq 0 \quad \text { in } \mathbb{R} \times \mathbb{R}_{+}, \quad \lim _{t \rightarrow \infty} v(\xi, t)=0 .
$$

Then the function

$$
w(\rho, t):=v(\rho-c t, t) \quad(\rho \geq 0, t \geq 0) .
$$

satisfies the problem

$$
\begin{cases}\frac{\partial w}{\partial t}=\frac{\partial^{2} w}{\partial \rho^{2}}+(n-1) \frac{\partial w}{\partial \rho}+f(w) & \text { in } \mathbb{R}_{+} \times \mathbb{R}_{+}, \\ w=\varphi & \text { in } \mathbb{R}_{+} \times\{0\}\end{cases}
$$

and

$$
\frac{\partial w}{\partial \rho} \leq 0 \quad \text { in } \mathbb{R}_{+} \times \mathbb{R}_{+}
$$

Since coth $\rho \geq 1$, by (5.1)-(5.2) $w$ is a supersolution of problem (4.2). 
Then by comparison results and inequality (4.3) we have

$$
u(x, t) \leq \tilde{u}(\rho(x), t) \leq w(\rho(x), t)=v(\rho(x)-c t, t) \quad \text { in } \mathbb{H}^{n} \times \mathbb{R}_{+} .
$$

Let $y \in \mathbb{H}^{n}$. Since $\xi \mapsto v(\xi, t)$ is nonincreasing for any $t>0$, and $d(x, y)>c t$ implies $\rho(x)-c t>-\rho(y)$, by (4.9) we obtain

$$
\begin{aligned}
\sup _{\left\{x \in \mathbb{H}^{n} \mid d(x, y)>c t\right\}} u(x, t) & \leq \sup _{\left\{x \in \mathbb{H}^{n} \mid d(x, y)>c t\right\}} v(\rho(x)-c t, t) \\
& \leq v(-\rho(y), t) \rightarrow 0 \text { as } t \rightarrow \infty .
\end{aligned}
$$

Hence the claim follows.

(ii) Under the present assumptions equality (3.5) holds,

$$
\lim _{t \rightarrow \infty} u(x, t)=1 \quad \text { uniformly on compact subsets of } \mathbb{H}^{n} .
$$

Therefore, for any compact $K \subseteq \mathbb{H}^{n}$ and for any $\eta \in(0,1)$ there exists $h=h(K, \eta)>0$ such that

$$
u(x, t) \geq \eta \quad \text { for any } x \in K, t \geq h .
$$

Let $c \in\left(0, c_{0}-(n-1)\right)$. Fix $\bar{c} \in\left(c, c_{0}-(n-1)\right)$ and $\bar{R}>0$ such that

$$
\operatorname{coth} \bar{R}<1+\frac{\bar{c}-c}{n-1}
$$

thus in particular

$$
\operatorname{coth} \bar{R}<1+\frac{\bar{c}}{n-1} .
$$

Let $\gamma_{\bar{c}} \in(0,1), \bar{\eta} \in\left(\gamma_{\bar{c}}, 1\right), \bar{b}=b(\bar{c}, \bar{\eta})>0, \bar{q}, \bar{v}, \bar{v}_{0}$ denote the quantities used in the proof of Lemma 4.2, with $c$ replaced by $\bar{c}$ and $R$ by $\bar{R}$. Then by Lemma 4.2 we have

$$
\bar{v}(x, t) \geq \bar{\eta} \quad \text { if } \rho(x) \leq \bar{R}+\bar{k} t,
$$

where $\bar{v}$ denotes the solution of problem (4.21) with Cauchy data $\bar{v}_{0}$ and

$$
\bar{k} \equiv k(\bar{c}):=\bar{c}+(n-1)(1-\operatorname{coth} \bar{R})>0
$$

(see (4.23)-(4.24)).

On the other hand, observe that the definition of $\bar{v}_{0}$ (see (4.20)) and the inequality $\bar{q}^{\prime}<0$ in $(0, \bar{b}]$ imply

$$
\bar{v}_{0}(\rho(x)) \leq \bar{\eta} \quad \text { if } \rho(x) \leq \bar{R}+\bar{b}, \quad \bar{v}_{0}(\rho(x))=0 \quad \text { otherwise. }
$$

Therefore, using inequality (5.3) in the compact set $K=\left\{x \in \mathbb{H}^{n} \mid \rho(x) \leq \bar{R}+\bar{b}\right\}$ we obtain

$$
u(x, h) \geq \bar{v}_{0}(\rho(x)) \quad \text { for any } x \in \mathbb{H}^{n},
$$

whence by comparison results

$$
u(x, t+h) \geq \bar{v}(x, t) \quad \text { for any }(x, t) \in \mathbb{H}^{n} \times \mathbb{R}_{+} .
$$


From (5.5)-(5.6) we obtain

$u(x, t+h) \geq \bar{\eta} \quad$ for any $(x, t) \in \mathbb{H}^{n} \times \mathbb{R}_{+}$such that $\rho(x) \leq \bar{R}+\bar{k} t \quad\left(t \in \mathbb{R}_{+}\right)$,

that is,

$u(x, t) \geq \bar{\eta} \quad$ for any $(x, t) \in \mathbb{H}^{n} \times \mathbb{R}_{+}$such that $\rho(x) \leq \bar{R}+\bar{k}(t-h) \quad(t>h)$.

Let $y \in \mathbb{H}^{n}$. Since inequality (5.4) implies $c<\bar{k}$, we have

$$
\left\{x \in \mathbb{H}^{n} \mid d(x, y)<c t\right\} \subseteq\left\{x \in \mathbb{H}^{n} \mid \rho(x)<\bar{R}+\bar{k}(t-h)\right\}
$$

for any $t \in \mathbb{R}_{+}$sufficiently large. Therefore, by the above remarks

$$
\inf _{d(x, y)<c t} u(x, t) \geq \inf _{\rho(x) \leq \bar{R}+\bar{k}(t-h)} u(x, t) \geq \bar{\eta} .
$$

To summarize, we have proved that for any $\bar{\eta} \in\left(\gamma_{\bar{c}}, 1\right)$ there exists $\tau \in \mathbb{R}_{+}$such that

$$
\inf _{d(x, y)<c t} u(x, t) \geq \bar{\eta} \quad \text { for any } t>\tau .
$$

Since $u \leq 1$ in $\mathbb{H}^{n} \times \mathbb{R}_{+}$, equality (3.5) follows.

Proof of Theorem 3.7. Denote by $\omega$ the convex hull of the support of $u_{0}$. Take any hyperplane $\pi \subseteq \mathbb{H}^{n}$ such that $\pi \cap \omega=\emptyset$. Let $\mathbb{H}_{\omega}^{n} \subseteq \mathbb{H}^{n}$ be the half-space containing $\omega$. Define

$$
\tilde{u}(x):=u\left(R_{\pi}(x)\right) \quad\left(x \in \mathbb{H}^{n}\right),
$$

where $R_{\pi}$ denotes the reflection in $\pi$. Observe that both $u$ and $\tilde{u}$ satisfy the equation

$$
\frac{\partial u}{\partial t}=\Delta_{H} u+f(u) \quad \text { in } \mathbb{H}_{\omega}^{n} \times \mathbb{R}_{+},
$$

whereas

$$
u=u_{0} \geq 0=\tilde{u} \quad \text { in } \mathbb{H}_{\omega}^{n} \times\{0\}, \quad u=\tilde{u} \quad \text { in } \pi \times \mathbb{R}_{+} .
$$

Then by the strong maximum principle we get

$$
u>\tilde{u} \quad \text { in } \mathbb{H}_{\omega}^{n} \times \mathbb{R}_{+},
$$

and by the Hopf Boundary Lemma we obtain

$$
\frac{\partial u}{\partial v}>\frac{\partial \tilde{u}}{\partial v}=-\frac{\partial u}{\partial v} \quad \text { in } \pi \times \mathbb{R}_{+},
$$

where $v$ is the vector field orthogonal to $\pi$ pointing towards $\mathbb{H}_{\omega}^{n}$. Hence

$$
\left\langle\nabla_{H} u, v\right\rangle_{H}>0 \quad \text { in } \pi \times \mathbb{R}_{+} .
$$

(i) Let us now prove the first claim. Since supp $u_{0}$ is compact and (3.5) holds, we have $\Gamma_{a}(u ; t) \cap \omega=\emptyset$ for any $a \in(0,1)$ and $t \in \mathbb{R}_{+}$sufficiently large. Let $x_{0} \in \Gamma_{a}(u ; t)$. Then we can find a hyperplane $P \subseteq \mathbb{H}^{n}$ such that $x_{0} \in P$ and $P \cap \omega=\emptyset$ (see Remark 2.1 (ii)). Using the above remarks with $\pi=P$, by (5.7) we obtain $\nabla_{H} u\left(x_{0}, t\right) \neq 0$. Since $x_{0}$ is arbitrary, the smoothness of $\Gamma_{a}(u ; t)$ follows. 
(ii) To prove the second claim we assume for contradiction that there exist $x_{0} \in$ $\Gamma_{a}(u ; t)$ and an infinite geodesic $\gamma$ orthogonal to $\Gamma_{a}(u ; t)$ at $x_{0}$, which does not intersect $\omega$. Then we can choose a hyperplane $Q \subseteq \mathbb{H}^{n}$ such that $\gamma \subseteq Q$ and $Q \cap \omega$ $=\varnothing$ (see Remark 2.1(iii)). Using the above remarks with $\pi=Q$, by (5.7) we obtain $\left\langle\nabla_{H} u\left(x_{0}, t\right), \tau\right\rangle_{H} \neq 0$, where $\tau$ is a tangent vector to $\Gamma_{a}(u ; t)$ in $x_{0}$. However, this contradicts the very definition of $\Gamma_{a}(u ; t)$. Hence the conclusion follows.

\section{Horospheric waves: proofs}

Proof of Theorem 3.9. Since the function $u$ defined in (1.18) only depends on $\left(x_{n}, t\right)$, plainly we have

$$
\begin{aligned}
\frac{\partial u}{\partial t}-\Delta_{H} u-f(u) & =-c^{*}\left(q^{*}\right)^{\prime}-\left[\left(q^{*}\right)^{\prime \prime}+\left(q^{*}\right)^{\prime}\right]+(2-n)\left(q^{*}\right)^{\prime}-f\left(q^{*}\right) \\
& =-\left(q^{*}\right)^{\prime \prime}+\left(1-n-c^{*}\right)\left(q^{*}\right)^{\prime}-f\left(q^{*}\right)=0
\end{aligned}
$$

(here the expression (2.17) of $\Delta_{H}$ in $\mathbb{U}^{n}$ has been used). Therefore $u$ satisfies equation (1.12). Moreover, its level set is

$$
\Gamma_{a}(u ; t):=\left\{x \in \mathbb{U}^{n} \mid x_{n}=e^{-z-c^{*} t}\right\} \quad\left(a \in(0,1), t \in \mathbb{R}_{+}\right),
$$

where $z:=\left(q^{*}\right)^{-1}(a)$ (recall that $q^{*}$ is decreasing in $\mathbb{R}$ by Proposition 3.1(i)). Then for any $a \in(0,1)$ and $t \in \mathbb{R}_{+}, \Gamma_{a}(u ; t)$ is a horosphere with center at infinity. This completes the proof.

To prove Theorem 3.10 we need some preliminary results. As already remarked, the expression (1.18) of a horospheric wave in $\mathbb{U}^{n}$ is formally analogous to that of a planar wave in $\mathbb{R}^{n}$. This analogy suggests expressing solutions of problem (3.12) in a moving frame, so that horospheric waves appear as stationary states - namely, to introduce the new coordinate $e^{c^{*} t} x_{n}\left(t \in \mathbb{R}_{+}\right)$. However, in contrast to the case of translations in $\mathbb{R}^{n}$, the change of coordinates

$$
\chi_{t}: \mathbb{U}^{n} \rightarrow \mathbb{U}^{n}, \quad \chi_{t}\left(x^{\prime}, x_{n}\right):=\left(x^{\prime}, e^{c^{*} t} x_{n}\right) \quad\left(x^{\prime} \in \mathbb{R}^{n-1}, x_{n}>0, t \in \mathbb{R}_{+}\right)
$$

is not an isometry of $\mathbb{U}^{n}$ (hereafter, any point $x \in \mathbb{U}^{n}$ is denoted by $x \equiv\left(x^{\prime}, x_{n}\right)$ with $\left.x^{\prime} \equiv\left(x_{1}, \ldots, x_{n-1}\right) \in \mathbb{R}^{n-1}, x_{n} \in \mathbb{R}_{+}\right)$. Instead, the map

$$
\tau_{t}: \mathbb{U}^{n} \rightarrow \mathbb{U}^{n}, \quad \tau_{t}\left(x^{\prime}, x_{n}\right):=e^{c^{*} t}\left(x^{\prime}, x_{n}\right) \equiv\left(X^{\prime}, X_{n}\right) \quad\left(t \in \mathbb{R}_{+}\right)
$$

is an isometry (see (2.16)). Therefore we define

$$
w:=u \circ \tau_{t}^{-1}: \mathbb{U}^{n} \times \mathbb{R}_{+} \rightarrow \mathbb{R}, \quad w\left(X^{\prime}, X_{n}, t\right):=u\left(e^{-c^{*} t} X^{\prime}, e^{-c^{*} t} X_{n}, t\right) \quad\left(t \in \mathbb{R}_{+}\right),
$$

where $u=u\left(x^{\prime}, x_{n}, t\right)$ is any solution of problem (3.12). An elementary calculation shows that $w$ solves the problem

$$
\left\{\begin{array}{l}
\frac{\partial w}{\partial t}=X_{n}^{2} \Delta w-\left(c_{0}-1\right) \frac{\partial w}{\partial X_{n}} X_{n}-c^{*} \sum_{i=1}^{n-1} \frac{\partial w}{\partial X_{i}} X_{i}+f(w) \quad \text { in } \mathbb{U}^{n} \times \mathbb{R}_{+}, \\
w\left(X^{\prime}, X_{n}, 0\right)=w_{0}\left(X^{\prime}, X_{n}\right):=u_{0}\left(X^{\prime}, X_{n}\right)
\end{array}\right.
$$

$\left(\left(X^{\prime}, X_{n}\right) \in \mathbb{U}^{n}\right.$; here the expression (2.17) of $\Delta_{H}$ in $\mathbb{U}^{n}$ has been used). 
Define $z:=-\log X_{n}$ and

$$
v: \mathbb{R}^{n} \times \mathbb{R}_{+} \rightarrow \mathbb{R}, \quad v\left(X^{\prime}, z, t\right):=w\left(X^{\prime}, e^{-z}, t\right) .
$$

Then problem (6.1) reads

$$
\left\{\begin{array}{l}
\frac{\partial v}{\partial t}=\frac{\partial^{2} v}{\partial z^{2}}+c_{0} \frac{\partial v}{\partial z}+e^{-2 z} \sum_{i=1}^{n-1} \frac{\partial^{2} v}{\partial X_{i}^{2}}-c^{*} \sum_{i=1}^{n-1} \frac{\partial v}{\partial X_{i}} X_{i}+f(v) \quad \text { in } \mathbb{R}^{n} \times \mathbb{R}_{+}, \\
v\left(X^{\prime}, z, 0\right)=v_{0}\left(X^{\prime}, z\right):=w_{0}\left(X^{\prime}, e^{-z}\right)
\end{array}\right.
$$

$\left(\left(X^{\prime}, z\right) \in \mathbb{R}^{n}\right.$; recall that by definition $\left.c_{0}=c^{*}+n-1\right)$.

If $v$ does not depend on $X^{\prime} \in \mathbb{R}^{n-1}$, by (6.2) it satisfies the equation

$$
\frac{\partial v}{\partial t}=\frac{\partial^{2} v}{\partial z^{2}}+c_{0} \frac{\partial v}{\partial z}+f(v) \quad \text { in } \mathbb{R} \times \mathbb{R}_{+} .
$$

Concerning (6.3), let us recall the following lemma ([9]; see also [16, Lemma 4.2]).

Lemma 6.1. Let assumption $\left(H_{5}\right)$ be satisfied. Then for any $\delta_{1} \in(0, a)$ and any $\delta_{2} \in$ $(0,1-a)$ there exist $\beta>0$ and $C \geq 1$ (only depending on $\delta_{1}, \delta_{2}$ and $f$ ) such that the functions $v^{ \pm}: \mathbb{R} \times \mathbb{R}_{+} \rightarrow \mathbb{R}$

$$
\begin{aligned}
& v^{+}(z, t):=q^{*}\left(z-C \delta_{1}\left(1-e^{-\beta t}\right)\right)+\delta_{1} e^{-\beta t}, \\
& v^{-}(z, t):=q^{*}\left(z+C \delta_{2}\left(1-e^{-\beta t}\right)\right)-\delta_{2} e^{-\beta t}
\end{aligned}
$$

are respectively a supersolution and a subsolution of equation (6.3).

By a suitable modification of the proof of [16, Lemma 4.1], we can prove the following result.

Proposition 6.2. Let assumption $\left(H_{5}\right)$ be satisfied. Let $v=v\left(X^{\prime}, z, t\right)$ be any solution of problem (6.2).

(i) Suppose that

$$
\limsup _{z \rightarrow \infty} \sup _{X^{\prime} \in \mathbb{R}^{n-1}} v_{0}\left(X^{\prime}, z\right)<a .
$$

Then there exists $z^{*} \in \mathbb{R}$ such that

$$
\limsup _{t \rightarrow \infty} \sup _{X^{\prime} \in \mathbb{R}^{n-1}} v\left(X^{\prime}, z, t\right) \leq q^{*}\left(z-z^{*}\right) \quad \text { uniformly for } z \in \mathbb{R} .
$$

(ii) Similarly, if

$$
\liminf _{z \rightarrow-\infty} \inf _{X^{\prime} \in \mathbb{R}^{n-1}} v_{0}\left(X^{\prime}, z\right)>a,
$$

there exists $z_{*} \in \mathbb{R}$ such that

$$
\liminf _{t \rightarrow \infty} \inf _{X^{\prime} \in \mathbb{R}^{n-1}} v\left(X^{\prime}, z, t\right) \geq q^{*}\left(z-z_{*}\right) \quad \text { uniformly for } z \in \mathbb{R} \text {. }
$$


Proof. We only prove claim (i), the proof of (ii) being similar. To this end, we shall prove the following

Claim. There exist $\tau \in \mathbb{R}_{+}, z_{0} \in \mathbb{R}$ and $\delta_{1} \in(0, a)$ such that

$$
\sup _{X^{\prime} \in \mathbb{R}^{n-1}} v\left(X^{\prime}, z, \tau\right) \leq v^{+}\left(z-z_{0}, 0\right)=q^{*}\left(z-z_{0}-C \delta_{1}\right)+\delta_{1} \quad \text { for any } z \in \mathbb{R} .
$$

By the Claim the conclusion follows. In fact, inequality (6.9) and Lemma 6.1 yield by comparison results

$$
\sup _{X^{\prime} \in \mathbb{R}^{n-1}} v\left(X^{\prime}, z, t\right) \leq v^{+}\left(z-z_{0}, t-\tau\right) \quad \text { for any } z \in \mathbb{R}, t \geq \tau .
$$

Letting $t \rightarrow \infty$ in the above inequality gives

$$
\limsup _{t \rightarrow \infty} \sup _{X^{\prime} \in \mathbb{R}^{n-1}} v\left(X^{\prime}, z, t\right) \leq q^{*}\left(z-z_{0}-C \delta_{1}\right)
$$

(see (6.4)), which is inequality (6.6) with $z^{*}:=z_{0}+C \delta_{1}$.

It remains to prove the Claim. This will be done in three steps.

(i) By inequality (6.5) we can choose $\delta_{1} \in(0, a)$ and $\beta \in\left(0, \delta_{1}\right)$ such that

$$
\limsup _{z \rightarrow \infty} \sup _{X^{\prime} \in \mathbb{R}^{n-1}} v_{0}\left(X^{\prime}, z\right)<\beta<\delta_{1} .
$$

Let us show that for every fixed $\tau \in \mathbb{R}_{+}$,

$$
\limsup _{z \rightarrow \infty} \sup _{X^{\prime} \in \mathbb{R}^{n-1}} v\left(X^{\prime}, z, \tau\right)<\delta_{1} .
$$

To this end, choose first $M \geq 1$ such that

$$
\sup _{X^{\prime} \in \mathbb{R}^{n-1}} v_{0}\left(X^{\prime}, z\right) \leq \beta+M e^{-c_{0} z} \quad \text { for any } z \in \mathbb{R} .
$$

This is possible, since by inequality (6.10) there exists $\bar{z}>0$ such that

$$
\sup _{X^{\prime} \in \mathbb{R}^{n-1}} v_{0}\left(X^{\prime}, z\right) \leq \beta \quad \text { for any } z>\bar{z}
$$

moreover,

$$
\sup _{X^{\prime} \in \mathbb{R}^{n-1}} v_{0}\left(X^{\prime}, z\right) \leq 1 \leq e^{-c_{0}(z-\bar{z})} \quad \text { for any } z \leq \bar{z} .
$$

Setting $M:=e^{c_{0} \bar{z}}$ we obtain (6.12).

Further, let us observe that the function

$$
\bar{v}(z, t):=\beta+M e^{-c_{0}(z-\alpha t)} \quad\left(z \in \mathbb{R}, t \in \mathbb{R}_{+}\right)
$$

is a supersolution of the first equation in (6.2) for any $\alpha>0$ sufficiently large. In fact, it is easily checked that

$$
\frac{\partial \bar{v}}{\partial t}-\frac{\partial^{2} \bar{v}}{\partial z^{2}}-c_{0} \frac{\partial \bar{v}}{\partial z}-f(\bar{v})=\alpha M e^{-c_{0}(z-\alpha t)}-f\left(\beta+M e^{-c_{0}(z-\alpha t)}\right) \geq 0
$$


if $\alpha \geq \frac{1}{a-\beta} \sup _{s \in[a, 1]} f(s)$, since

$$
f(\bar{v}) \geq 0 \Leftrightarrow 0 \leq z-\alpha t \leq \frac{1}{c_{0}}\left|\log \frac{a-\beta}{M}\right|
$$

(see assumption $\left(H_{5}\right)$ ).

By (6.12)-(6.13) the function $\bar{v}$ (with $\alpha, \beta, M$ as above) is a supersolution of problem (6.2). Therefore by comparison we obtain

$$
\sup _{X^{\prime} \in \mathbb{R}^{n-1}} v\left(X^{\prime}, z, \tau\right) \leq \beta+M e^{-c_{0}(z-\alpha \tau)}
$$

for any $z \in \mathbb{R}$ and any fixed $\tau \in \mathbb{R}_{+}$. Hence inequality (6.11) follows.

(ii) Since by assumption $\left(H_{5}\right)$ we have $f(s)<0$ if $s>1$, the function $\tilde{v}:=1+\delta_{1} / 2$ is a supersolution of problem (6.2). Hence by comparison

$$
\sup _{X^{\prime} \in \mathbb{R}^{n-1}} v\left(X^{\prime}, z, \tau\right) \leq 1+\delta_{1} / 2 \quad \text { for any } z \in \mathbb{R}, \tau \in \mathbb{R}_{+} .
$$

(iii) Fix any $\tau \in \mathbb{R}_{+}$. Set $z_{1}:=C \delta_{1}+\left(q^{*}\right)^{-1}\left(1-\delta_{1} / 2\right)$. Then by (6.14) we have

$$
\sup _{X^{\prime} \in \mathbb{R}^{n-1}} v\left(X^{\prime}, z, \tau\right) \leq 1+\delta_{1} / 2 \leq q^{*}\left(z-C \delta_{1}\right)+\delta_{1} \quad \text { for any } z \geq z_{1} .
$$

On the other hand, by (6.11) there exists $z_{2}>0$ such that

$$
\sup _{X^{\prime} \in \mathbb{R}^{n-1}} v\left(X^{\prime}, z, \tau\right) \leq \delta_{1} \quad \text { for any } z>z_{2} .
$$

Defining $z_{0}:=\max \left\{z_{2}-z_{1}, 0\right\}$ we obtain inequality (6.9), thus the Claim follows.

Proof of Theorem 3.10. Under the change of variables $\left(x^{\prime}, x_{n}\right) \rightarrow\left(X^{\prime}, z\right)$, assumption (3.13) corresponds to assumptions (6.5) and (6.7) of Proposition 6.2. Then by inequalities (6.6) and (6.8) we obtain

$$
q^{*}\left(z-z_{*}\right) \leq v\left(X^{\prime}, z, t\right) \leq q^{*}\left(z-z^{*}\right) \quad \text { for every }\left(X^{\prime}, z\right) \in \mathbb{R}^{n}, t \in \mathbb{R}_{+} .
$$

Since by definition $z=-\log x_{n}-c^{*} t$ and $v\left(X^{\prime}, z, t\right)=u\left(x^{\prime}, x_{n}, t\right)$, setting $k^{*}:=e^{z^{*}}$ and $k_{*}:=e^{z_{*}}$ yields inequality (3.14).

\section{References}

[1] Ahlfors, L. A.: Möbius Transformations in Several Variables. Lecture Notes, School of Mathematics, Univ. of Minnesota (1981) Zbl 0517.30001 MR 0725161

[2] Aronson, D. G., Weinberger, H. F.: Nonlinear diffusion in population genetics, combustion, and nerve pulse propagation. In: Partial Differential Equations and Related Topics, Lecture Notes in Math. 446, Springer, 6-49 (1975) Zbl 0325.35050 MR 0427837

[3] Aronson, D. G., Weinberger, H. F.: Multidimensional nonlinear diffusion arising in population genetics. Adv. Math. 30, 33-76 (1978) Zbl 0407.92014 MR 0511740 
[4] Bandle, C., Pozio, M. A., Tesei, A.: The Fujita exponent for the Cauchy problem in the hyperbolic space. J. Differential Equations 251, 2143-2163 (2011) Zbl 1227.35052 MR 2823663

[5] Benedetti, R., Petronio, C.: Lectures on Hyperbolic Geometry. Springer (1992) Zbl 0768.51018 MR 1219310

[6] Berestycki, H., Hamel, F.: Generalized travelling waves for reaction diffusion equations. In: Perspectives in Nonlinear Partial Differential Equations. In Honor of H. Brezis, Contemp. Math. 446, Amer. Math. Soc., 101-123 (2007) Zbl 1200.35169 MR 2373726

[7] Davies, E. B.: Heat Kernel and Spectral Theory. Cambridge Univ. Press (1989) MR 0990239

[8] Fenchel, W.: Elementary Geometry in Hyperbolic Space. De Gruyter (1989) Zbl 0674.51001 MR 1004006

[9] Fife, P. C., McLeod, J. B.: The approach of solutions of nonlinear diffusion equations to travelling front solutions. Arch. Ration. Mech. Anal. 65, 335-361 (1977) Zbl 0361.35035 MR 0442480

[10] Fujita, H.: On the blowing up of solutions of the Cauchy problem for $u_{t}=\Delta u+u^{1+\alpha}$. J. Fac. Sci. Tokyo Sect. IA Math. 13, 109-124 (1966) Zbl 0163.34002 MR 0214914

[11] Grigoryan, A.: Analytic and geometric background of recurrence and non-explosion of the Brownian motion on Riemannian manifolds. Bull. Amer. Math. Soc. 36, 135-249 (1999) Zbl 0927.58019 MR 1659871

[12] Iversen, B.: Hyperbolic Geometry. London Math. Soc. Student Texts 25, Cambridge Univ. Press (1992) Zbl 0766.51002 MR 1205776

[13] Jones, C.: Asymptotic behaviour of a reaction-diffusion equation in higher space dimensions. Rocky Mountain J. Math. 13, 355-364 (1983) Zbl 0528.35054 MR 0702830

[14] Kumaresan, S., Prajapat, J.: Serrin's result for hyperbolic space and sphere. Duke Math. J. 91, 17-28 (1998) Zbl 0941.35029 MR 1487977

[15] Kumaresan, S., Prajapat, J.: Analogue of Gidas-Ni-Nirenberg result in hyperbolic space and sphere. Rend. Ist. Mat. Univ. Trieste 30, 107-112 (1998) Zbl 0930.35072 MR 1704819

[16] Matano, H., Nara, M.: Large time behavior of disturbed planar fronts in the Allen-Cahn equation. J. Differential Equations 251, 3522-3557 (2011) Zbl 1252.35050 MR 2837694

[17] Pozio, M. A., Punzo, F., Tesei, A.: Uniqueness and nonuniqueness of solutions to parabolic problems with singular coefficients. Discrete Contin. Dynam. Systems 30, 891-916 (2011) Zbl 1232.35011 MR 2784626 